\title{
STOCHASTIC PROCESSES AND ANTIDERIVATIONAL EQUATIONS ON NON-ARCHIMEDEAN MANIFOLDS
}

\author{
S. V. LUDKOVSKY
}

Received 24 December 2002

\begin{abstract}
Stochastic processes on manifolds over non-Archimedean fields and with transition measures having values in the field $\mathbb{C}$ of complex numbers are studied. Stochastic antiderivational equations (with the non-Archimedean time parameter) on manifolds are investigated.
\end{abstract}

2000 Mathematics Subject Classification: 28C20, 46 S10.

1. Introduction. Stochastic processes and stochastic differential equations on real Banach spaces and manifolds on them were intensively studied (see, e.g., [2, 4, 5, $12,13,14,34,35,36,39]$ and the references therein). The stochastic processes considered there were with values in either real Banach spaces or manifolds on them. The results of these investigations were used in many mathematical and theoretical physical problems. In particular, stochastic processes on some Lie groups were studied. On the other hand, the development of non-Archimedean functional analysis, non-Archimedean quantum physical theories and quantum mechanics poses problems of developing measure theory, and stochastic processes on non-Archimedean Banach spaces and manifolds on them $[15,16,17,37,38,40]$. Some steps in this direction were made in $[1,3,7,8,9,10,19,41]$. In those articles, real-valued and complex-valued stochastic processes were considered, also stochastic processes with values in nonArchimedean fields and linear spaces over them, but with compact or locally compact supports of transition measures, were considered. There, pseudodifferential stochastic equations based on pseudodifferential operators in the sense of Vladimirov [40] were also considered. These pseudodifferential operators are quite different from antiderivational operators of Schikhof [37]. The latter serve as the non-Archimedean analog of the indefinite integration, while the former serve as non-Archimedean analogs of the classical pseudodifferential operators. There can be different variants of nonArchimedean stochastic processes, depending on whether the time parameter is either non-Archimedean or real, a space is of functions either complex-valued or with values in a non-Archimedean field. Then transition measures may be either complex-valued or with values in a non-Archimedean field. It totally gives eight variants. The case of the non-Archimedean time and a space of functions with values in a non-Archimedean space was not practically considered in $[1,3,7,8,9,10,19,41]$. The present paper is devoted to the latter variant and its meaning is primarily in its applicability for investigations of unitary representations of totally disconnected nonlocally compact groups. It also permits the construction of volume elements associated with transition measures 
on an infinite-dimensional over non-Archimedean field manifolds, quasi-invariant relative to the corresponding diffeomorphism group. Then such stochastic processes on manifolds can be applied in the non-Archimedean quantum field theory and gauge theory. The above-cited papers do not permit considering the quasi-invariant transition measures of stochastic processes and then such measures on nonlocally compact spaces or groups.

In preceding works of the author, measures and stochastic processes on nonArchimedean Banach spaces and totally disconnected topological groups with values in non-Archimedean spaces were investigated [25, 26, 29, 30, 31, 32, 22, 23, 27]. Quasiinvariant measures on groups and manifolds were used for investigations of their representations [21, 24, 28].

In this paper, non-Archimedean stochastic processes and stochastic antiderivational equations (with the non-Archimedean time parameter) on manifolds on Banach spaces over non-Archimedean fields are investigated. Moreover, wider classes of stochastic processes are considered in this work than in preceding works of the author [30, 31, 32, 33]. Analogs of Lèvy processes were studied in [1, 8, 9, 19, 20, 41]. In [20], specific classes of non-Archimedean Gaussian-type measures and Wiener-type processes were defined and investigated, having more properties analogous to the classical case than in preceding works. In this paper, Itô bundles on non-Archimedean Banach manifolds are defined and investigated. For this specific transformation, formulas of stochastic processes, which were not studied by other authors, are proved.

It is necessary to note that in this paper not only manifolds treated by the rigid geometry are considered, but also much wider classes. For them, the existence of an exponential mapping is proved. A rigid non-Archimedean geometry serves mainly for needs of the cohomology theory on such manifolds, but it is too restrictive and operates with narrow classes of analytic functions [11]. It was introduced at the beginning of the sixties of the 20th century. Few years later, wider classes of functions were investigated by Schikhof [37]. In this paper, classes of functions and antiderivation operators by Schikhof, and their generalizations from the works [25, 26, 23], are used. The contents of this paper do not intersect with the previous works cited above.

The results of this paper permit the consideration of stochastic processes on nonArchimedean manifolds as well as more general classes of stochastic processes on non-Archimedean Banach spaces and totally disconnected topological groups. Some other principal differences of the classical and non-Archimedean stochastic analyses are discussed in [30, 31, 32, 33]. All results of this paper are obtained for the first time.

2. Stochastic processes on non-Archimedean manifolds. To avoid misunderstanding, definitions and notations are given at first.

2.1. Definitions and notes. Let $M$ be a $C^{n}$-manifold on a Banach space $X$ over a nonArchimedean field $\mathbf{K}$ complete relative to its norm with an atlas $\operatorname{At}(M):=\left\{\left(U_{j}, \phi_{j}\right): j \in\right.$ $\left.\Lambda_{M}\right\}$, such that $U_{j}$ is an open covering of $M, \phi_{j}: U_{j} \rightarrow \phi_{j}\left(U_{j}\right)$ is a homeomorphism, $\phi_{j}\left(U_{j}\right)$ is open in $X, \phi_{l} \circ \phi_{j}^{-1}: \phi_{j}\left(U_{j} \cap U_{l}\right) \rightarrow \phi_{l}\left(U_{j} \cap U_{l}\right)$ is a diffeomorphism of class $C^{n}$ for each $U_{j} \cap U_{l} \neq \varnothing$, and the space $C^{n}(U, Y)$ of functions from an open subset 
$U \subset X$ into a Banach space $Y$ over $\mathbf{K}$ is defined in terms of difference quotients (see $[25,26,37])$.

Since the derivative $\left(\phi_{l} \circ \phi_{j}^{-1}\right)^{\prime}(x)$ is a linear continuous operator on $\phi_{j}\left(U_{j} \cap U_{l}\right) \times X$ of class $C^{n-1}$ for each $n \geq 1$, and there exists a derivative of an inverse operator $\left(\phi_{j} \circ \phi_{l}^{-1}\right)^{\prime}(y)$ on $\phi_{l}\left(U_{j} \cap U_{l}\right) \times X$, then $\left(\phi_{l} \circ \phi_{j}^{-1}\right)^{\prime}(x) \in \mathrm{GL}(X)$ for each $x \in \phi_{j}\left(U_{j} \cap\right.$ $\left.U_{l}\right)$, where $\mathrm{GL}(X)$ is the group of invertible $\mathbf{K}$-linear bounded operators of $X$ onto $X$. Therefore, for each $n \geq 1$, there exists a functor $T$ such that $T\left(\phi_{l} \circ \phi_{j}^{-1}\right)(x):=$ $\left(\phi_{l, j}(x), \phi_{l, j}^{\prime}(x)\right)$ for each $x \in\left(\phi_{l} \circ \phi_{j}^{-1}\right)\left(U_{j} \cap U_{l}\right), T\left(\phi_{j}\left(U_{j}\right)\right):=\phi_{j}\left(U_{j}\right) \times X$, where $\phi_{l, j}:=\left(\phi_{l} \circ \phi_{j}^{-1}\right)$. For $n \geq 1$, put $T M=\bigcup_{j \in \Lambda_{M}} T U_{j}$ with the atlas $\operatorname{At}(T M):=\left\{\left(U_{j} \times\right.\right.$ $\left.\left.X, T \phi_{j}\right): j \in \Lambda_{M}\right\}$, such that $T \phi_{j}: T U_{j} \rightarrow \phi_{j}\left(U_{j}\right) \times X$ is a homeomorphism, $\left.T \phi_{j}\right|_{\{x\} \times X}=$ : $T_{x} \phi_{j}$ is a bounded continuous operator on $X$ by the second argument for each $x \in U_{j}$. Thus $T M=\bigcup_{x \in M} T_{x} M$, where $T_{x} \phi_{j}: T_{x} U_{j} \rightarrow T_{\phi_{j}(x)} \phi_{j}\left(U_{j}\right)$ is a $\mathbf{K}$-linear isomorphism for each $j \in \Upsilon_{M}$, where $T_{\phi_{j}(x)} \phi_{j}\left(U_{j}\right)=\left\{\phi_{j}(x)\right\} \times X$. TM is called the total tangent space of $M, T_{X} M$ is called the tangent space of $M$ at $x$. The projection $\tau:=\tau_{M}: T M \rightarrow M$ is given by $\tau_{M}(s)=x$ for each vector $s \in T_{X} M, \tau_{M}$ is called the tangent bundle.

2.1.1. If $M$ and $N$ are two $C^{l}$-manifolds on Banach spaces $X$ and $S$ over $\mathbf{K}$ with $l \geq n$, where $\operatorname{At}(N):=\left\{\left(V_{j}, \psi_{j}\right): j \in \Lambda_{N}\right\}$, and $f: M \rightarrow N$ is a continuous mapping, then by definition, $f \in C^{n}(M, N)$, if $\psi_{l} \circ f \circ \phi_{j}^{-1} \in C^{n}\left(W_{l, j}, Y\right)$ for each $W_{l, j}:=\phi_{j}\left(f^{-1}\left(V_{l}\right) \cap\right.$ $\left.U_{j}\right) \neq \varnothing$. A norm in $C^{n}(X, S)$ induces a complete uniformity in $C^{n}(M, N)$. If $n \geq 1$ and $f \in C^{n}(M, N)$, then there exists $T f: T M \rightarrow T N$ and $T f \in C^{n-1}(T M, T N)$.

2.1.2. Let $H$ and $X$ be two Banach spaces over a non-Archimedean field $\mathbf{K}$. Let $M$ be a $C^{l}$-manifold on $X$ and let $P$ be a manifold with a mapping $\pi: P \rightarrow M$ such that $\pi$ is surjective and $\pi^{-1}(x)=: P_{x}=: H_{x}$ is a Banach space over $\mathbf{K}$ isomorphic to $H$ for each $x \in M, \pi$ is called a projection, $\pi^{-1}(x)$ is called a fibre of $\pi$ over $x$. Suppose that $P$ is supplied with an atlas $\operatorname{At}(P)=\left\{\left(U_{j}, \phi_{j}, P \phi_{j}\right): j \in \Lambda_{M}\right\}$ consistent with $\operatorname{At}(M)$, such that $\mathrm{pr}_{1} \circ P \phi_{j}=\left.\phi_{j} \circ \pi\right|_{P U_{j}}$ on $\pi^{-1}\left(U_{j}\right)$ for each $j$, where $\operatorname{pr}_{1}: U_{j} \times H \rightarrow U_{j}$ and $\mathrm{pr}_{2}: U_{j} \times H \rightarrow H$ are projections, $P \phi_{j}$ is bijective, $P_{x} \phi_{j}=\left.P \phi_{j}\right|_{H_{x}}: H_{x} \rightarrow\left\{\phi_{j}(x)\right\} \times H$ is a Banach space isomorphism, $P \phi_{l} \circ\left(\left.P \phi_{j}\right|_{P\left(U_{l} \cap U_{j}\right)}\right)^{-1}: \phi_{j}\left(U_{j} \cap U_{l}\right) \times H \rightarrow \phi_{l}\left(U_{j} \cap U_{l}\right) \times H$ is a $C^{n}$-diffeomorphism, $l \geq n$. Two atlases are called equivalent if their union is an atlas. $(P, M, \pi)$ is called a vector bundle over $M$ with fibre on $H . P$ is called the total space of $\pi$, and $M$ the base space of $\pi$.

Let $\left(P_{1}, M_{1}, \pi_{1}\right)$ and $\left(P_{2}, M_{2}, \pi_{2}\right)$ be two vector bundles with spaces $H_{1}$ and $H_{2}$ for the fibres of $\pi_{1}$ and $\pi_{2}$, respectively. Suppose that there are two $C^{n}$-mappings $F: M_{1} \rightarrow M_{2}$ and $P F: P_{1} \rightarrow P_{2}$ such that $\pi_{2} \circ P F=F \circ \pi$ on $P_{1}$ and the restriction $P_{x} F:=\left.P F\right|_{H_{1, x}}$ : $H_{1, x} \rightarrow H_{2, F(x)}$ is a K-linear mapping. Then $(F, P F)$ is called a morphism from $\left(P_{1}, M_{1}, \pi_{1}\right)$ to $\left(P_{2}, M_{2}, \pi_{2}\right)$.

2.1.3. A $C^{m}$-vector field on $M$ is a $C^{m}$-mapping $\Psi: M \rightarrow T M$ such that $\tau_{M} \circ \Psi=$ id. If $F: M \rightarrow N$ is a $C^{m}$-morphism and $\Psi: M \rightarrow T N$ is such that $\tau_{N} \circ \Psi=F$, then $\Psi$ is called a vector field along $F$.

Suppose that $\mathbf{K}$ is spherically complete, then a topologically adjoint space $H^{*}$ of $\mathbf{K}$ linear functionals on a Banach space $H$ over $\mathbf{K}$ separates points of $H, H^{*} \neq \varnothing$ (see [38, Lemma 4.3.5]). The bundle of $r$-fold contravariant and $s$-fold covariant tensors over $M$ is defined by $L\left(\tau^{*}, \ldots, \tau^{*}, \tau, \ldots, \tau ; \rho\right): L\left(T^{*} M, \ldots, T^{*} M, T M, \ldots, T M ; \mathbf{K} M\right) \rightarrow M$ or shortly 
$\tau_{s}^{r}: T_{s}^{r} M \rightarrow M$, where $\tau^{*}$ and $T^{*} M$ are repeated $r$ times, $\tau$ and $T M$ are repeated $s$ times, and $\rho: \mathbf{K} M=M \times \mathbf{K} \rightarrow M$ is the trivial bundle over $M$. Here, $L\left(\alpha_{1}, \ldots, \alpha_{r} ; \beta\right): L\left(A_{1}, \ldots, A_{r} ; B\right)$ $\rightarrow M$ denotes a vector bundle over $M$, where $\left(A_{j}, M, \alpha_{j}\right)$ and $(B, M, \beta)$ are vector bundles, $\alpha_{k}^{-1}(x)=H_{k, x}, k=1, \ldots, r, \beta_{H}^{-1}(x)=Y_{x}, L\left(A_{1}, \ldots, A_{r} ; B\right):=\bigcup_{x \in M} L\left(H_{1, x}, \ldots, H_{r, x} ; Y_{x}\right)$, $H_{k, x}$ and $Y_{x}$ are isomorphic to Banach spaces $H_{k}$ and $Y$, respectively, over K. For each chart $\left(U_{j}, \phi_{j}\right)$ of $M$, the bundle chart $\left(U_{j}, \phi_{j}, L\left(A_{1}, \ldots, A_{r} ; B\right) \phi_{j}\right)$ is given by

$$
L\left(A_{1} \phi_{j}(x), \ldots, A_{r} \phi_{j}(x) ; B \phi_{j}(x)\right): L\left(H_{1, x}, \ldots, H_{r, x} ; Y_{x}\right) \longrightarrow L\left(H_{1}, \ldots, H_{r} ; Y\right),
$$

such that for $\Psi_{x} \in L\left(H_{1, x}, \ldots, H_{r, x} ; Y_{x}\right)$, its image is

$$
L\left(A_{1} \phi_{j}(x), \ldots ; B \phi_{j}(x)\right) \Psi_{x}=B \phi_{j}(x) \circ \Psi_{x} \circ\left(A_{1} \phi_{j}(x)^{-1} \times \cdots \times A_{r} \phi_{j}(x)^{-1}\right),
$$

$A_{k} \phi_{j}(x)^{-1}: H_{k, x} \rightarrow H_{k}$ is the $\mathbf{K}$-linear isomorphism of Banach spaces as well as $B \phi_{j}(x)$ : $Y \rightarrow Y_{x}, L\left(H_{1}, \ldots, H_{r} ; Y\right)$ is the Banach space of all continuous mappings $f: H_{1} \times \cdots \times$ $H_{r} \rightarrow Y$ such that $f$ is $\mathbf{K}$-linear by each variable $z_{k} \in H_{k}, k=1, \ldots, r$.

If $\Psi: M \rightarrow \kappa T M$ is a $C^{m}$-mapping, such that $\kappa \circ \Psi=i d$, then $\Psi$ is called a tensor field (of type $\kappa$ ), where $(\kappa T M, M, \kappa(\tau))$ is a tensor bundle over $M$. If $(P, N, \pi)$ is a vector bundle and $F: M \rightarrow N$ is a morphism, then a morphism $\theta: M \rightarrow P$, with $\pi \circ \theta=F$, is called a section along $F$.

2.1.4. Let $M$ be a $C^{n}$-manifold on a Banach space $X$ over a spherically complete nonArchimedean field $\mathbf{K}$ and let $\mathscr{B}_{n} M$ denote the set of all $C^{n}$-vector fields on $M$, where $n \geq 2$. Let $\Gamma={ }_{j} \Gamma: \phi_{j}\left(U_{j}\right) \ni y_{j} \mapsto \Gamma\left(y_{j}\right) \in L(X, X ; X)$ be a $C^{n-2}$-mapping such that

$$
\phi_{l, j}^{\prime} \cdot{ }_{j} \Gamma\left(y_{j}\right)=\phi_{l, j}{ }^{\prime \prime}+{ }_{l} \Gamma\left(y_{l}\right) \circ\left(\phi_{l, j}^{\prime} \times \phi_{l, j}^{\prime}\right),
$$

for each two charts with $U_{j} \cap U_{l} \neq \varnothing$. This $\left\{{ }_{j} \Gamma\right\}$ is called the family of Christoffel symbols ${ }_{j} \Gamma$ on $M$.

A covariant derivation $\mathscr{B}_{n-1} M^{2} \ni(\Psi, \Phi) \mapsto \nabla_{\Psi} \Phi \in \mathscr{B}_{n-2} M$ is given by

$$
\nabla_{\Psi} \Phi\left(y_{j}\right)=\Phi^{\prime}\left(y_{j}\right) \cdot \Psi\left(y_{j}\right)+\Gamma\left(y_{j}\right)\left(\Psi\left(y_{j}\right), \Phi\left(y_{j}\right)\right)
$$

where $\Psi\left(y_{j}\right)$ and $\Phi\left(y_{j}\right)$ are principal parts of $\Psi$ and $\Phi$ on $\left(U_{j}, \phi_{j}\right)$. If $M$ with $\operatorname{At}(M)$ is supplied with $\Gamma$, then $M$ possesses a covariant derivation.

2.1.5. For a $C^{n}$-vector bundle $(P, M, \pi)$ on $X \times H$, with $n \geq 2$, define a $\mathbf{K}$-(linear) connection as a bundle morphism $K: T P \rightarrow P$ such that $\pi \circ K=\pi \circ \tau_{P}$. This mapping $K$ in its local representation ${ }_{j} K=P \phi_{j} \circ K \circ T P \phi_{j}^{-1}$ for bundle charts $\left(U_{j}, \phi_{j}, P \phi_{j}\right)$ of $(P, M, \pi)$ and $\left(T U_{j}, T \phi_{j}, T P \phi_{j}\right)$ of $\left(T P, P, \tau_{P}\right)$ is given by $\left\{U_{j}, \Xi\right\} \times(X \times H) \ni(x, \Psi, \Phi, z) \mapsto(x, z+$ $\left.{ }_{j} \Gamma(x)(\Phi, \Psi)\right) \in\{x\} \times H$. The Christoffel symbol ${ }_{j} \Gamma(x): U_{j} \rightarrow L(X, H ; H)$ is of class of smoothness $C^{n-2}$. For it, the horizontal space $T_{\Psi h}$ is defined as the kernel of $\left.K\right|_{T_{\Psi} P}$ : $T_{\Psi} P \rightarrow H_{q}, q=\pi(\Psi)$.

For a section $\Psi: M \rightarrow P$ in $(P, M, \pi)$, define the covariant derivation of $\Psi$ in the direction $\Phi \in T_{x} M$ by

$$
\nabla_{\Phi} \Psi(x)=K \circ T_{\chi} \Psi \cdot \Phi
$$


2.2. Let $X$ be either finite-dimensional over a local field $\mathbf{K}$ space or of countable type such that a sequence of subspaces $S_{n}$ is given by $S_{n} \subset S_{n+1}$ and $S_{n} \neq S_{n+1}$ for each $n \in \mathbb{N}, \operatorname{cl}\left(\bigcup_{n} S_{n}\right)=X$, and a dimension $\operatorname{dim}_{\mathbf{K}} S_{n}=: m(n)$ of $S_{n}$ over $\mathbf{K}$ is finite. Let $U$ be a clopen bounded subset in $S_{n}$. Consider an antiderivation operator $P(l, s)$ on the Banach space $C((t, s-1), U \rightarrow \mathbf{K})$ of functions $f: U \rightarrow \mathbf{K}$ with definite partial difference quotients having continuous extensions (see [25, 26, Section I.2]), and denote $P(l, s)$ on $U$ by $P_{U}(l, s)$, where $t \in[0, \infty), 1 \leq s \in \mathbb{Z}, l=[t]+1$, [t] is an integer part of $t$. In particular, $C((t, 0), U \rightarrow \mathbf{K})$ is denoted here by $C^{t}(U, \mathbf{K})$.

2.3. Definition and note. Now let $U$ be a clopen bounded subset in $X$ with $\operatorname{dim}_{\mathbf{K}} X=$ $\infty$. For each $f \in C_{0}((t, s-1), U \rightarrow \mathbf{K})$, there exists a sequence of cylindrical functions $f_{n}$, such that $f=\sum_{n} f_{n}$ and $\lim _{n}\left\|\hat{f}_{n}\right\|_{C\left((t, s-1), U_{n} \rightarrow \mathbf{K}\right)}=0$, where $f_{n}$ is a cylindrical function on $U$ such that $f_{n}(x)=\hat{f}_{n}\left(\pi_{n} x\right), \hat{f}_{n}$ is a function on $U_{n}:=S_{n} \cap U, \pi_{n}: X \rightarrow S_{n}$ is a projection on $S_{n}$. For each $t<\infty$, there exists $U$ of sufficiently small diameter $\delta$ such that $\left\|P_{U_{n}}(l, s)\right\| \leq 1$ for each $n$, since it is sufficient to take $\delta^{|j|+n} /|(j+\bar{u}) !| \leq 1$ for each $j$ with $\left|j^{\prime}\right|=0, \ldots, l-1, j=j^{\prime}+s^{\prime} \bar{u}, s^{\prime} \in\{0,1, \ldots, s-1\}$ (see [25, 26, Definition I.2.11]). For $U$ of $\operatorname{diam}(U)$ satisfying such condition, define $P_{U}(l, s) f:=\sum_{n} P_{U_{n}}(l, s) f_{n}$.

For $U$ as above, the space ${ }_{P} C_{0}((t, s), U \rightarrow Y):=Y \oplus P_{U}(l, s) C_{0}((t, s-1), U \rightarrow Y)$, where $Y$ is a Banach space over $\mathbf{K}$, is defined as well as ${ }_{P}^{2} C_{0}((t, s), U \rightarrow Y):=Y \oplus$ $P_{U}(l, s)_{P} C_{0}((t, s-1), U \rightarrow Y)$ for $s \geq 2$.

LEMMA 2.1. An image $P_{U}(t, s)(C((t, s-1), U \rightarrow Y))$ denoted by ${ }_{P, 0} C((t, s), U \rightarrow Y)$ is contained in $C((t, s), U \rightarrow Y)$ and does not coincide with the latter space. The space ${ }_{P, 0} C((t, s), U \rightarrow Y)$ can be supplied with a norm denoted by $\|*\|_{U,(t, s), P}$, relative to which it is complete, and $P_{U}(l, s):\left(C((t, s-1), U \rightarrow Y),\|*\|_{C((t, s-1), U \rightarrow Y)}\right) \rightarrow\left({ }_{P, 0} C((t, s), U \rightarrow\right.$ $\left.Y),\|*\|_{U,(t, s), P}\right)$ is continuous.

Proof. First, consider $\operatorname{dim}_{\mathbf{K}} X<\infty$. If $f \in{ }_{P, 0} C((t, s), U \rightarrow Y)$, then $\partial^{\bar{u}}(P(t, s) f)(x)=$ $f(x)$ for each $x \in U$ (see [25, 26, Corollary I.2.16]). On the other hand, there is $g \in$ $C((t, s), U \rightarrow Y)$ for which $\partial^{e_{j}} g(x)=0$ in the notation of [25, 26, Definitions 2.4.1 and $2.11 \mathrm{I}$.$] , for example, locally constant g$.

Now let $X$ be infinite-dimensional, then from taking the limit of $f_{n}$, this statement follows in the general case. Consider an image $P_{U}(l, s)(B(C((t, s-1), U \rightarrow Y), 0,1))=: V$ of the closed ball in $C((t, s), U \rightarrow Y)$ containing 0 and with the unit radius. Let $f \in$ ${ }_{P} C((t, s), U \rightarrow Y)$, then there exists $g \in C((t, s-1), U \rightarrow Y)$, such that $P_{U}(l, s) g=f$. On the other hand, $\|g\|_{C((t, s-1), U \rightarrow Y)}<\infty$ and there exists a constant $0 \neq c \in \mathbf{K}$, such that $c g \in B(C((t, s-1), U \rightarrow Y), 0,1)$. Therefore, $c f \in V$, since $P_{U}(l, s)$ is the $\mathbf{K}$-linear operator, that is, $V$ is the absorbing subset. Since the ball $B(C((t, s-1), U \rightarrow Y), 0,1)$ is $\mathbf{K}$-convex, then $V$ is K-convex. Evidently, $0 \in V$.

Consider a weak topology on $C((t, s), U \rightarrow Y)$, then it induces a weak topology on its K-linear subspace ${ }_{P} C((t, s), U \rightarrow Y)$. In particular, each evaluation functional $h_{x}(f):=$ $f(x)$ is $\mathbf{K}$-linear and continuous on the latter space, where $x \in U$. In view of [25, 26, Theorem I.2.15], $P_{U}(l, s)$ is continuous from $C((t, s-1), U \rightarrow Y) \rightarrow C((t, s), U \rightarrow Y)$. Therefore, $V$ is bounded-relative to the weak topology, since $U$ is compact and $V$ is bounded-relative to a weaker topology generated by evaluation functionals. Let $\eta$ be a Minkowski functional on ${ }_{P, 0} C((t, s), U \rightarrow Y)$ generated by $V$. It generates a norm in 
${ }_{P, 0} C((t, s), U \rightarrow Y)$, relative to which it is complete. Since $V$ is the unit ball relative to this norm and $P_{U}(l, s)^{-1}(V)$ is the unit ball in $C((t, s-1), U \rightarrow Y)$, then $P_{U}(l, s)$ is continuous relative to this topology.

NoTE 2.2. In view of Lemma 2.1, the definitions in Sections 2.1.1, 2.1.2, 2.1.3, 2.1.4, and 2.1.5 can be spread on $C_{0}((t, s))$ and ${ }_{P}^{2} C_{0}((t, s))$ manifolds, that is, $\left(\phi_{l, j}-\mathrm{id}\right) \in$ $C_{0}((t, s))$ and $\left(\phi_{l, j}-\mathrm{id}\right) \in{ }_{P}^{2} C_{0}\left((t, s), W_{l, j} \rightarrow X\right)$, respectively, for each charts $U_{l}$ and $U_{j}$ with $U_{l} \cap U_{j} \neq \varnothing$, where $\phi_{j}\left(U_{j}\right)$ are bounded clopen subsets in $X$ of sufficiently small diameter, as in Section 2.3, if $X$ is infinite-dimensional over $\mathbf{K}$.

Note 2.3. Consider the space of functions $\mathscr{F}_{(t, s)} M=C_{0}((t, s), M \rightarrow \mathbf{K})$, then $\nabla_{S}(a V+$ $b W)=a \nabla_{S} V+b \nabla_{S} W, \nabla_{S}(f V)=S(f) V+f \nabla_{S} V$, where $S, V, W \in \mathscr{B}_{(t, s)} M$, and $\mathscr{B}_{(t, s)} M$ denotes the set of all $C_{0}((t, s))$-vector fields on $M$. Considering the foliation of $M$ and taking the limit, we get for a given chart $\left(U_{j}, \phi_{j}\right)$,

$$
\nabla_{S} V\left(\phi_{j}\right)=\sum_{k}\left\{\sum_{i} S^{i}\left(\phi_{j}\right)\left(\frac{\partial V^{k}}{\partial \phi_{j}^{i}}\right)\left(\phi_{j}\right)+\sum_{i, l} S^{i}\left(\phi_{j}\right) V^{l}\left(\phi_{j}\right) \Gamma_{i, l}^{k}\left(\phi_{j}\right)\right\} e_{k},
$$

where $\left(\phi_{j}, e_{i}\right)$ are basic vector fields on $\phi_{j}\left(U_{j}\right), S\left(\phi_{j}\right)=\sum_{i} S^{i}\left(\phi_{j}\right) e_{i}, \Gamma\left(\phi_{j}\right)=$ $\sum_{i, l, k} \Gamma_{i, l}^{k}\left(\phi_{j}\right) e^{i} \otimes e^{l} \otimes e_{k}, e^{i}\left(e_{j}\right)=\delta_{j}^{i}$ for each $i$ and $j \in \alpha$. Therefore, there exist a torsion tensor $T(S, V)=\nabla_{S} V-\nabla_{V} S-[S, V]$ and a curvature tensor $R(S, V) W=\nabla_{S} \nabla_{V} W-$ $\nabla_{V} \nabla_{S} W-\nabla_{[S, V]} W$ for each $S, V$ and $W \in \mathscr{B}_{(t, s)} M$, such that $T(S, V)=-T(V, S), R(S, V) W$ $=-R(V, S) W$ and $T\left(\phi_{j}\right)(S, V)=\Gamma\left(\phi_{j}\right)(S, V)-\Gamma\left(\phi_{j}\right)(V, S), T\left(\phi_{j}\right) \in L(X, X ; X)$, $R\left(\phi_{j}\right)(S, V) W=D \Gamma\left(\phi_{j}\right) \cdot S(V, W)-D \Gamma\left(\phi_{j}\right) \cdot V(S, W)+\Gamma\left(\phi_{j}\right)\left(S, \Gamma\left(\phi_{j}\right)(V, W)\right)-$ $\Gamma\left(\phi_{j}\right)\left(V, \Gamma\left(\phi_{j}\right)(S, W)\right), R\left(\phi_{j}\right) \in L(X, X, X ; X)$ analogously to [18, Lemma 1.5.3].

THEOREM 2.4. Let $M$ be $a{ }_{P}^{2} C_{0}((t, s))$-manifold with $s \geq 2$, then there exist a clopen neighborhood $\tilde{T} M$ of $M$ in $T M$ and an exponential $C_{0}((t, s))$-mapping exp : $\tilde{T} M \rightarrow M$ of $\tilde{T} M$ on $M$.

Proof. Let $M$ be embedded into $T M$ as the zero-section of the bundle $\tau_{M}$. Consider the non-Archimedean geodesic equation $\nabla_{\dot{c}} \dot{c}=0$ with initial conditions $c(0)=x_{0}$, $\dot{c}(0)=y_{0}, x_{0} \in M, y_{0} \in T_{x_{0}} M$, where $c(b)$ is a ${ }_{P}^{2} C_{0}((t, s))$-curve on $M, c: B(\mathbf{K}, 0,1) \rightarrow M$. For a chart $\left(U_{j}, \phi_{j}\right)$ containing a point $x$ of $M$, let $\phi_{j} \circ c(b):=\psi_{j}(b)$, thus

$$
\psi_{j}^{\prime \prime}(b)+\Gamma\left(\psi_{j}(b)\right)\left(\dot{\psi}_{j}(b), \dot{\psi}_{j}(b)\right)=0 .
$$

Since $\psi_{j} \in{ }_{P}^{2} C_{0}((t, s))$, then there exists $f \in C((t, s-2), B \rightarrow X)$ such that $\psi_{j}=y_{1}+$ $P_{B}(l, s)\left(y_{2}+P_{B}(l, s-1) f\right)$, where $B:=B(\mathbf{K}, 0,1), y_{1}, y_{2} \in Y$. Therefore, $\dot{\psi}_{j}=y_{2}+P_{B}(l, s-$ 1) $f$ and $\psi_{j}{ }^{\prime \prime} s=f$; consequently, $f$ satisfies the equation

$$
f(b)+\Gamma\left(y_{1}+\left.P^{2}\left(y_{2}+P^{1} f\right)\right|_{b}\right)\left(y_{2}+\left.P^{1} f\right|_{b}, y_{2}+\left.P^{1} f\right|_{b}\right)=0,
$$

where $P^{2}:=P_{B}(l, s)$ and $P^{1}:=P_{B}(l, s-1)$. Consider a marked point $b_{0} \in B$. At first, there exists $r>0$ such that (2.8), and hence (2.7), has a unique solution in $B\left(\mathbf{K}, b_{0}, r\right)$. For this, consider the iterational equation

$$
f_{m+1}(b)+\Gamma\left(y_{1}+\left.P^{2}\left(y_{2}+P^{1} f_{m}\right)\right|_{b}\right)\left(y_{2}+\left.P^{1} f_{m}\right|_{b}, y_{2}+\left.P^{1} f_{m}\right|_{b}\right)=0,
$$


where $f_{m}$ is a sequence of functions. From $\Gamma \in C_{0}((t, s-2))$, since $M$ is the ${ }_{P}^{2} C_{0}((t, s))$ manifold, it follows that $f_{m+1} \in C((t, s-2))$ for each $f_{m} \in C((t, s-2))$. Then

$$
\begin{aligned}
f_{m+1}(t)-f_{m}(t)= & -\Gamma\left(y_{1}+\left.P^{2}\left(y_{2}+P^{1} f_{m}\right)\right|_{t}\right)\left(y_{2}+\left.P^{1} f_{m}\right|_{t}, y_{2}+\left.P^{1} f_{m}\right|_{t}\right) \\
& +\Gamma\left(y_{1}+\left.P^{2}\left(y_{2}+P^{1} f_{m}\right)\right|_{t}\right)\left(y_{2}+\left.P^{1} f_{m-1}\right|_{t}, y_{2}+\left.P^{1} f_{m-1}\right|_{t}\right) \\
& -\Gamma\left(y_{1}+\left.P^{2}\left(y_{2}+P^{1} f_{m}\right)\right|_{t}\right)\left(y_{2}+\left.P^{1} f_{m-1}\right|_{t}, y_{2}+\left.P^{1} f_{m-1}\right|_{t}\right) \\
& +\Gamma\left(y_{1}+\left.P^{2}\left(y_{2}+P^{1} f_{m-1}\right)\right|_{t}\right)\left(y_{2}+\left.P^{1} f_{m-1}\right|_{t}, y_{2}+\left.P^{1} f_{m-1}\right|_{t}\right) .
\end{aligned}
$$

In view of the ultrametric inequality, bilinearity of $\Gamma(x)(a, b)$ by $a, b$, continuity by $x$, and continuity of $P^{1}$ and $P^{2}$ for each $x_{0} \in M$ and each $t_{0} \in B(\mathbf{K}, 0,1)$, there exist $r>0$ and $\epsilon>0$ such that

$$
\left\|f_{m+1}-f_{m}\right\| \leq C \epsilon^{2}\|\Gamma\|\left\|f_{m}-f_{m-1}\right\| \text { for each } t \in B\left(\mathbf{K}, t_{0}, r\right) \text { and each }\left\|y_{0}\right\|<\epsilon,
$$

where $C>0$ is a constant related to $P^{1}$ and $P^{2}$. There exists $0<r<\infty$ such that $\left\|P^{1}\right\| \leq$ 1 , $\left\|P^{2}\right\| \leq 1$, and $P^{2} P^{1} f \in G_{j, k} \subset U_{j}$ for each $f \in G_{j, k}$, since $t$ and $s$ are finite (see above), where $G_{j, k}$ is a clopen subset in $U_{j},\|\Gamma\|$ is a norm of $\Gamma$ on $G_{j, k} \times X^{2}$ as a bilinear operator on $X$ for each $x \in G_{j, k}$. In view of continuity of $\Gamma$ and boundedness of $\phi_{j}\left(U_{j}\right)$ for each $j$, it is possible to choose a locally finite covering $G_{j, k}$ subordinated to $U_{j}$, such that $\|\Gamma\|$ is finite on $G_{j, k}, k \in \mathbb{N}$. Therefore, choosing $C \epsilon^{2}\|\Gamma\|<1$, we get a convergent sequence on $B\left(\mathbf{K}, t_{0}, r\right) \times G_{j, k} \times B(X, 0, \delta)$, and due to the fixed point theorem, there exists a unique solution in $B\left(\mathbf{K}, t_{0}, r\right)$. In view of compactness of $B(\mathbf{K}, 0,1)$, there exists a solution on it. Let $f$ and $g$ be two functions providing solutions $\psi^{f}=P^{2} P^{1} f$ and $\psi^{g}=P^{2} P^{1} g$ of the problem on $B(\mathbf{K}, 0,1)$, then $P^{2} P^{1} f\left(t_{l}\right)=P^{2} P^{1} g\left(t_{l}\right), P^{1} f\left(t_{l}\right)=P^{1} g\left(t_{l}\right)$ for a finite number of points $t_{0}=0, t_{1}, \ldots, t_{k} \in B(\mathbf{K}, 0,1)$, such that on each $B\left(\mathbf{K}, t_{j}, r_{j}\right)$, a solution is unique for a given initial condition, $0<r_{j} \leq 1$ for each $j$, and $\bigcup_{j} B\left(\mathbf{K}, t_{j}, r_{j}\right)=B(\mathbf{K}, 0,1)$. This implies that

$$
\begin{aligned}
& \Gamma\left(\left.P^{2}\left(y_{2}+P^{1} f\right)\right|_{t}\right)\left(y_{2}+\left.P^{1} f\right|_{t}, y_{2}+\left.P^{1} f\right|_{t}\right) \\
& \quad-\Gamma\left(\left.P^{2}\left(y_{2}+P^{1}\left(f+c_{1, l}\right)\right)\right|_{t}\right)\left(y_{2}+\left.P^{1}\left(f+c_{2, l}\right)\right|_{t}, y_{2}+\left.P^{1}\left(f+c_{2, l}\right)\right|_{t}\right)=c_{1, l}
\end{aligned}
$$

for each $l$ and each $t \in B\left(\mathbf{K}, t_{l}, r_{l}\right)$. On the other hand, $P^{1} c$ and $P^{2} c$ are not locally constant for a constant $c \neq 0, \Gamma\left(\phi_{j}\right)(a, b)$ is bilinear by $(a, b) \in X^{2}$ and satisfies (2.3), hence (2.12) may be satisfied only for $c_{1, l}=c_{2, l}=0$ for each $l$; consequently, a solution is unique.

Since $f \in C_{0}((t, s-2))$, then $\psi_{j} \in{ }_{P} C_{0}((t, s))$ for each $j$, since $\psi_{j}=y_{1}+P_{B}(l, s)\left(y_{2}+\right.$ $\left.P_{B}(l, s-1) f\right)$. Moreover, $c_{a S}(t)=c_{S}(a t)$ for each $a \in B(\mathbf{K}, 0,1)$ such that $\left|a S\left(\phi_{j}(q)\right)\right|<$ $\epsilon$, since $d c_{S}(a t) / d t=\left.a\left(d c_{S}(z) / d z\right)\right|_{z=a t}$. In view of continuity of $P^{2}$ and $P^{1}$ and $\Gamma$ operators, for each $x_{0} \in M$, there exist a chart $\left(U_{j}, \phi_{j}\right)$ and clopen neighborhoods $V_{1}$ and $V_{2}, \phi_{j}\left(x_{0}\right) \in V_{1} \subset V_{2} \subset \phi_{j}\left(U_{j}\right)$ and $\delta>0$, such that from $S \in T M$ with $\tau_{M} S=q \in$ $\phi_{j}^{-1}\left(V_{1}\right)$ and $\left|S\left(\phi_{j}(q)\right)\right|<\delta$, it follows that the geodesic $c_{S}$ with $c_{S}(0)=S$ is defined for each $t \in B(\mathbf{K}, 0,1)$ and $c_{S}(t) \in \phi_{j}^{-1}\left(V_{2}\right)$. Due to paracompactness of $T M$ and $M$, this covering can be chosen locally finite [6]. 
This means that there exists a clopen neighborhood $\tilde{T} M$ of $M$ in $T M$ such that a geodesic $c_{S}(t)$ is defined for each $S \in \tilde{T} M$ and each $t \in B(\mathbf{K}, 0,1)$. Therefore, define the exponential mapping $\exp : \tilde{T} M \rightarrow M$ by $S \mapsto c_{S}(1)$, denote by $\exp _{x}:=\left.\exp \right|_{\tilde{T} M \cap T_{X} M}$ a restriction to a fibre. Then exp has a local representation $\left(x_{0}, y_{0}\right) \in V_{1} \times B(X, 0, \delta) \mapsto$ $\psi_{j}\left(1 ; x_{0}, y_{0}\right) \in V_{2} \subset \phi_{j}\left(U_{j}\right)$. From (2.7) and (2.8), it follows that exp is of $C_{0}((t, s))$-class of smoothness from $\tilde{T} M$ onto $M$.

Corollary 2.5. If $M$ is $a_{P}^{2} C_{0}((t, s)) \cap C^{\infty}$-manifold with $s \geq 2$, then $\exp \in C^{\infty}(\tilde{T} M$, $M)$.

Note 2.6. If $M$ is an analytic manifold, then $\exp : \tilde{T} M \rightarrow M$ is a locally analytic mapping. Theorem 2.4 gives an exponential manifold mapping for a wider class of manifolds than that treated by the rigid geometry.

2.4. Note and definitions. Let $M$ be a $C^{\infty}$-manifold and let $\tau_{M}: T M \rightarrow M$ be the tangent bundle, $\theta: M \times H \rightarrow M$ a trivial bundle over $M$ with a Banach space fibre $H$ over K. There exists the bundle $L_{1, r}\left(\theta, \tau_{M}\right)$ over $M$ with the fibre $L_{1, r}(H, X)$, where $r \geq 1$, and the spaces $L_{n, r}(H, X)$ were defined in [27] and [31, Section 2].

Let $M$ be a $C^{\infty}$-manifold with functions $\phi_{l, j}$ satisfying [31, Conditions (4.13)]. Suppose that $w$ is a stochastic process with values in $H$, and $\xi$ a stochastic process with values in $X$, such that $\lambda\left\{\omega: w(t, \omega) \in C^{0} \backslash C^{1}\right\}=0$, where $H$ and $X$ are Banach spaces over a local field $\mathbf{K}$ (see [31, Definition 4.1]; here $X$ has another meaning than $X$ in [31, 4.1 Remark and definition]). Let $a \in L^{q}\left(\Omega, \mathscr{F}, \lambda ; C^{0}\left(B_{R}, L^{q}\left(\Omega, \mathscr{F}_{F}, \lambda ; C^{0}\left(B_{R}, X\right)\right)\right)\right)$ and $E \in$ $L^{r}\left(\Omega, \mathscr{F}, \lambda ; C^{0}\left(B_{R}, L\left(L^{q}\left(\Omega, \mathscr{F}, \lambda ; C^{0}\left(B_{R}, H\right)\right), L^{q}\left(\Omega, \mathscr{F}, \lambda ; C^{0}\left(B_{R}, X\right)\right)\right)\right)\right)$,

$$
\xi(t, \omega)=\xi_{0}(\omega)+\left.\left(\hat{P}_{u} a\right)(u, \omega, \xi)\right|_{u=t}+\left.\left(\hat{P}_{w(u, \omega)} E\right)(u, \omega, \xi)\right|_{u=t},
$$

where $1 \leq r, s, q \leq \infty, 1 / r+1 / s=1 / q, w \in L^{s}\left(\Omega, \mathscr{F}, \lambda ; C_{0}^{0}\left(B_{R}, H\right)\right), \xi \in L^{q}\left(\Omega, \mathscr{F}, \lambda ; C^{0}\left(B_{R}\right.\right.$, $X)$ ). Since $H$ and $X$ are isomorphic to $c_{0}\left(\alpha_{H}, \mathbf{K}\right)$, and $c_{0}\left(\alpha_{X}, \mathbf{K}\right)$, then $L_{n, r}(X, H)$ has the embedding into $L_{n, r}(H, H)$ for $\alpha_{X} \subset \alpha_{H}$, and $L_{n, r}(H, H)$ has an embedding into $L_{n, r}(X, X)$ for $\alpha_{H} \subset \alpha_{X}$. Inclusions Range $(E) \subset X$, Range $(w) \subset H$, and Range $(\xi) \subset X$ reduce this case to [30, Theorem 3.3]. In view of [31, Lemma 2.2 and formula (4.14)],

$$
d \phi(\xi(t, \omega))=J(\phi, a, E) a d t+J(\phi, a, E) E d w
$$

where

$$
J(\phi, a, E):=\left.\sum_{m=0}^{\infty}[m !]^{-1} \sum_{l=0}^{m}\left(\begin{array}{c}
m \\
l
\end{array}\right) \hat{P}_{u^{l}, w^{m-l}} \phi^{(m+1)} \circ\left(a^{\otimes l} \otimes E^{\otimes(m-l)}\right)\right|_{u=t} .
$$

For stochastic processes of type (i) in Theorem 3.4 [30], it is necessary to consider the following generalization of [31, Theorem 4.7].

Note 2.7. Consider $a \in L^{\infty}\left(\Omega, \mathscr{F}, \lambda ; C^{0}\left(B_{R}, L^{q}\left(\Omega, \mathscr{F}, \lambda ; C^{0}\left(B_{R}, X\right)\right)\right)\right)$ and $E \in L^{\infty}(\Omega, \mathscr{F}$, $\left.\lambda ; C^{0}\left(B_{R}, L\left(L^{q}\left(\Omega, \mathscr{F}, \lambda ; C^{0}\left(B_{R}, H\right)\right), L^{q}\left(\Omega, \mathscr{F}, \lambda ; C^{0}\left(B_{R}, X\right)\right)\right)\right)\right), a=a(t, \omega, \xi), E=E(t, \omega, \xi)$, $t \in B_{R}, \omega \in \Omega, \xi \in L^{q}\left(\Omega, \mathscr{F}_{F}, \lambda ; C^{0}\left(B_{R}, X\right)\right), \xi_{0} \in L^{q}(\Omega, \mathscr{F}, \lambda ; X), w \in L^{\infty}\left(\Omega, \mathscr{F}, \lambda ; C_{0}^{0}\left(B_{R}, H\right)\right)$, $1 \leq q \leq \infty$, where $a$ and $E$ satisfy the local Lipschitz condition (see [30, Theorem 3.3, 
condition (LLC)]). Suppose that $\xi$ is a stochastic process of type

$$
\begin{aligned}
\xi(t, \omega)= & \xi_{0}(\omega) \\
& +\left.\sum_{m+b=1}^{\infty} \sum_{l=0}^{m}\left(\hat{P}_{u^{b+m-l}, w(u, \omega)^{l}}\left[a_{m-l+b, l}(u, \xi(u, \omega)) \circ\left(I^{\otimes b} \otimes a^{\otimes(m-l)} \otimes E^{\otimes l}\right)\right]\right)\right|_{u=t},
\end{aligned}
$$

such that $a_{m-l, l} \in C^{0}\left(B_{R_{1}} \times B\left(L^{q}\left(\Omega, \mathscr{F}, \lambda ; C^{0}\left(B_{R}, X\right)\right), 0, R_{2}\right), L_{m}\left(X^{\otimes m} ; X\right)\right)$ (continuous and bounded on its domain) for each $n, l, 0<R_{2}<\infty$, and

$$
\lim _{n \rightarrow \infty} \sup _{0 \leq l \leq n}\left\|a_{n-l, l}\right\|_{C^{0}\left(B_{R_{1}} \times B\left(L^{q}\left(\Omega, \mathscr{F}, \lambda ; C^{0}\left(B_{R}, X\right)\right), 0, R_{2}\right), L_{n}\left(X^{\otimes n}, X\right)\right)}=0
$$

for each $0<R_{1} \leq R$ when $0<R<\infty$, or for each $0<R_{1}<R$ when $R=\infty$, for each $0<R_{2}<\infty$.

Moreover, suppose that a function $f$ satisfies the conditions

$$
f(u, x) \in C^{\infty}(T \times H, X)
$$

and

$$
\lim _{n \rightarrow \infty} \max _{0 \leq l \leq n}\left\|\left(\bar{\Phi}^{n} f\right)\left(t, x ; h_{1}, \ldots, h_{n} ; \zeta_{1}, \ldots, \zeta_{n}\right)\right\|_{C^{0}\left(T \times B(\mathbf{K}, 0, r)^{l} \times B(H, 0,1)^{n-l} \times B\left(\mathbf{K}, 0, R_{1}\right)^{n-l}, X\right)}=0
$$

for each $0<R_{1}<\infty$, where $h_{j}=e_{1}, \zeta_{j} \in B(\mathbf{K}, 0, r)$ for variables corresponding to $t \in T=B\left(\mathbf{K}, t_{0}, r\right)$, and $h_{j} \in B(H, 0,1), \zeta_{j} \in B\left(\mathbf{K}, 0, R_{1}\right)$ for variables corresponding to $x \in H$.

Analogously, $a, E, a_{l, m}$ for $\xi$ with values in $M$ are considered, substituting $C^{0}\left(B_{R}, H\right)$ on $C^{0}\left(B_{R}, M\right)$.

THEOREM 2.8. If conditions (2.17) are satisfied, then (2.16) has the unique solution in $B_{R}$. If, in addition, conditions (2.18) and (2.19) are satisfied, then

$$
\begin{aligned}
f(t, \xi(t, \omega))= & f\left(t_{0}, \xi_{0}\right) \\
& +\sum_{m+b \geq 1,0 \leq m \in \mathbb{Z}, 0 \leq b \in \mathbb{Z}}((m+b) !)^{-1} \\
& \times \sum_{l_{1}, \ldots, l_{m}}\left(\begin{array}{c}
m+b \\
m
\end{array}\right)\left(\hat { P } _ { u ^ { b + m - l } , w ( u , \omega ) ^ { l } } \left[\left(\partial^{(m+b)} f / \partial u^{b} \partial x^{m}\right)(u, \xi(u, \omega))\right.\right. \\
& \circ\left(a_{l_{1}, n_{1}} \otimes \cdots \otimes a_{l_{m}, n_{m}}\right) \\
& \left.\left.\circ\left(I^{\otimes b} \otimes a^{\otimes(m-l)} \otimes E^{\otimes l}\right)\right]\right)\left.\right|_{u=t},
\end{aligned}
$$

where $l_{1}+\cdots+l_{m}=m+b-l, n_{1}+\cdots+n_{m}=l, l_{1}, \ldots, l_{m}, n_{1}, \ldots, n_{m}$ are nonnegative integers.

Proof. The first part of the theorem follows from [30, 3.4] and embeddings of Section 2.4. Since $\sigma_{n} \circ \sigma_{m}(t)=\sigma_{n} \circ \sigma_{m+j}(t)$ for each $n \geq m, j>0$, and $\sigma_{0}(t)=t_{0}$, 
then from [31, Formula (2.4)], it follows that

$$
\begin{aligned}
\left.\hat{P}_{u^{l+b}, w} m a_{l+b, m} \circ\left(I^{\otimes b} \otimes a^{\otimes l} \otimes E^{\otimes m}\right)\right|_{u=t_{n}} ^{u=t_{n+1}}= & a_{l+b, m}\left(t_{n}\right) \\
& \circ\left(\left(t_{n+1}-t_{n}\right)^{\otimes b} \otimes\left(a\left(t_{n}\right)\left(t_{n+1}-t_{n}\right)\right)^{\otimes l}\right. \\
& \left.\otimes\left(E\left(t_{n}\right)\left(w\left(t_{n+1}\right)-w\left(t_{n}\right)\right)\right)^{\otimes m}\right),
\end{aligned}
$$

where other arguments are omitted for shortening the notation. Therefore, the second part of this theorem follows from [31, Formulas (4.15)] and (2.16).

Note 2.9. Let conditions (2.16), (2.17) (2.18), and (2.19) be satisfied and $\phi=f$ independent of $t$. Then, due to [31, Lemma 2.2] and Theorem 2.8 above, formula (2.14) is valid with new operator $J$,

$$
J(\phi, a, E):=\sum_{m=0}^{\infty}[m !]^{-1} \sum_{l_{1}, \ldots, l_{m}} \hat{P}_{u^{l}, w^{m-l}} \phi^{(m+1)} \circ\left(a_{l_{1}, n_{1}}, \ldots, a_{l_{m}, n_{m}}\right) \circ\left(a^{\otimes l} \otimes E^{\otimes(m-l)}\right),
$$

where $l_{1}+\cdots+l_{m}=l, n_{1}+\cdots+n_{m}=m-l$.

DEFINITION 2.10. Let $(\Pi, M, \pi)$ be a bundle on a manifold $M$ with fibres $X \oplus L(H, X)$ for each $x \in M$ and with transition functions $J(\phi, a, E):(a, E) \mapsto(J(\phi, a, E) a, J(\phi, a$, $E) E$ ), where $\phi=\phi_{j, l}$ for each pair of charts $\left(U_{j}, \phi_{j}\right)$ and $\left(U_{l}, \phi_{l}\right)$ with $U_{j} \cap U_{l} \neq \varnothing$, $a \in X, E \in L(H, X), J(\phi, a, E)$ is given by either (2.15) or (2.22).

2.5. Definition and note. Let $t \in T \subset \mathbf{K}$, where $\mathbf{K}$ is a local field and $T$ is clopen in $\mathbf{K}$. Let also $\left(U_{j}, \phi_{j}\right)$ be a chart of a manifold $M$ on a Banach space $X$ over $\mathbf{K}, x \in U_{j} \subset M$, $(a, E) \in \pi^{-1}(x)$ (see Definition 2.10). By $\varphi_{x}(a, E)$ is denoted a collection of $M$-valued stochastic processes $\xi$, such that $\xi \in U_{j}$ with probability 1 , where $\phi_{j} \circ \xi$ is a solution of either (2.13) or (2.16) for each $j$. Then, $\mathscr{G}_{x}(a, E)$ is called the germ of the diffusion process at the point $x$ defined by a pair $(a, E)$. It is, in addition, with a given family of sections $a_{l, m}$ of bundles $\left(\Pi_{l+m}, M, \pi_{l+m}\right)$ with fibres $L_{m+l}\left(X^{\otimes m+l} ; X\right)$, such that $a_{l, m, x} \in$ $\pi_{l+m}^{-1}(x)$ in Note 2.7. Therefore, Section 2.4 is the particular case of Note 2.7.

A section $U$ of the vector bundle $(\Pi, M, \pi)$ is the non-Archimedean analog of Itô's field over $M$.

THEOREM 2.11. Let $\phi$ and $\psi$ be two functions satisfying-conditions of either Section 2.4 or Note 2.7 such that $\operatorname{Dom}(\phi) \supset \operatorname{Range}(\psi)$. Then

$$
\begin{gathered}
J_{\psi(x)}(\phi, a, E) \circ J_{x}(\psi, a, E)=J_{x}(\phi \circ \psi, a, E), \\
J_{x}(\mathrm{id}, a, E)=\mathrm{id} .
\end{gathered}
$$

Proof. Since $a_{l, m, x} \in L_{l+m}\left(X^{\otimes l+m} ; X\right)$, then $J_{x}(\phi, a, E) a_{l, m, x} \circ\left(a^{\otimes l} \otimes E^{\otimes m}\right)=a_{l, m, x} \circ$ $\left(\left(J_{x}(\phi, a, E) a\right)^{\otimes l} \otimes\left(J_{x}(\phi, a, E) E\right)^{\otimes m}\right)$ for each $0 \leq l, m \in \mathbb{Z}$, and $x \in M$, where $a=a_{x}$, $E=E_{x},\left(a_{x}, E_{x}\right) \in \pi^{-1}(x)$. Each of the derivatives $\phi^{(m)}$ and $\psi^{(m)}$ is an $m$-polylinear operator on $X$. Therefore, $(\phi \circ \psi)^{(m)}(x)=\sum_{l_{1}+\cdots+l_{b} \geq m, 1 \leq b \leq m} R_{b} \circ\left(Q_{l_{1}} \otimes \cdots \otimes Q_{l_{b}}\right)$, where 
$R_{b}$ and $Q_{l}$ are the $b$-linear and $l$-linear operators corresponding up to constant multipli-

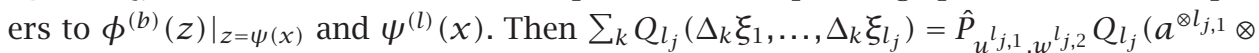
$\left.E^{\otimes l_{j, 2}}\right)$ for nonnegative $l_{j, 1}$ and $l_{j, 2}$ with $l_{j, 1}+l_{j, 2}=l_{j}$, and $\xi_{i}(t, \omega)=\left.\hat{P}_{u} a\right|_{u=t}$ for $i=$ $1, \ldots, l_{j, 1}, \xi_{i}(t, \omega)=\left.\hat{P}_{w} E\right|_{u=t}$ for $i=l_{j, 1}+1, \ldots, l_{j}$. Moreover, $\sum_{k} Q_{l_{j}}\left(\Delta_{k} \xi_{1}, \ldots, \Delta_{k} \xi_{l_{j}-1}\right.$,

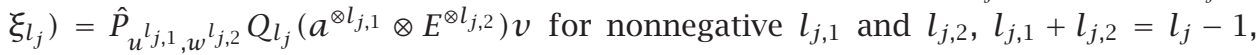
and $\xi_{i}(t, \omega)=\left.\hat{P}_{u} a\right|_{u=t}$ for $i=1, \ldots, l_{j, 1}, \xi_{i}(t, \omega)=\left.\hat{P}_{w} E\right|_{u=t}$ for $i=l_{j, 1}+1, \ldots, l_{j}-1$, $\xi_{l_{j}}=v$, where either $v=a$ or $v=E$. Therefore, $\phi: \mathscr{G}_{x}(a, E) \rightarrow \mathscr{G}_{\phi(x)}(J a, J E)$, where $J=J(\phi, a, E)$. In view of [31, Theorem 4.7] and Theorem 2.8, formulas (2.15) and (2.22) satisfy the equality

$$
J_{x}(\phi, a, E)=\phi^{\prime}\left(\xi_{x}^{0}\right)
$$

where $\xi^{0}$ is a stochastic process being the solution of either (2.13) or (2.16), $x \in M$, $\xi_{x}^{0} \in T_{x} M$. On the other hand, $(\phi(\psi)(x))^{\prime}=\phi^{\prime}(\psi(x)) \cdot \psi^{\prime}(x)$ for each $x \in \operatorname{Dom}(\psi)$. Therefore, from $\xi \in \operatorname{Dom}(\psi)$ and $\operatorname{Range}(\psi) \subset \operatorname{Dom}(\phi)$, formula (2.23) follows. From $\operatorname{id}^{\prime}=I$, where $I$ is a unit operator, formula (2.24) follows.

2.6. Remark and definition. Apart from the classical case, here the bundle associated with the operator $J(\phi, a, E)$ in general is not quadratic. It may be polynomial only in a particular case given by [31, Theorem 4.5].

Let $f=\exp$, where $\exp :=\exp ^{M}$ is the exponential mapping for $M$. Consider $\varphi_{(x, 0)}(a$, $E)$ a stochastic processes germ at a point $y=0$ in the tangent space $T_{x} M$. Then $\exp _{x}^{*} \mathscr{G}_{(x, 0)}(a, E)=\mathscr{G}_{x}\left(J\left(\exp _{x}, a, E\right)\right)(a, E)$ is a stochastic processes germ at $x \in M$. Therefore, $\phi_{j} \circ \exp _{x}^{*} \mathscr{G}_{(x, 0)}(a, E)=\mathscr{G}_{\phi(x)}\left(J\left(\phi_{j} \circ \exp _{x}, a, E\right)\right)(a, E)$ for each chart $\left(U_{j}, \phi_{j}\right)$ of $M$. The germ $\exp _{x}^{*} \varphi_{(x, 0)}(a, E)$ is called a stochastic differential bundle.

COROLLARY 2.12. To a functor $J$, there corresponds a bundle $\left(J^{M}, M, \pi_{J}\right)$, and a fibre $J_{x}^{M}:=\pi_{J}^{-1}(x)$ may be identified with the space $G_{x}\left(J^{M}\right)$ of stochastic processes germ. To a morphism $f: M \rightarrow N$ of manifolds, there corresponds a bundle morphism $G(f)=f * f^{*}$, where $f^{*} \xi:=f(\xi)$.

Proof. If $f: M \rightarrow N$ is a manifold morphism, then $U$ is transformed as $\left(a_{x}, E_{x}\right) \mapsto$ $\left(a_{f(x)}^{f}, E_{f(x)}^{f}\right)$, where $a_{f(x)}^{f}=J(f, a, E) a_{x}, E_{f(x)}^{f}=J(f, a, E) E_{x}$, and $a_{l, m, f(x)}^{f}\left(t, f^{*} \xi\right)=$ $a_{l, m, x}(t, \xi)$ for each $x \in M$. The stochastic process $\xi_{x}^{0}$ satisfies the antiderivational equation

$$
\xi_{x}^{0}=\sum_{l, m} \hat{P}_{u^{l}, w^{m}} a_{l, m, x} \circ\left(a_{x}^{\otimes l} \otimes E_{x}^{\otimes m}\right)
$$

and its differential has the form

$$
\begin{aligned}
d \xi_{x}^{0}= & \sum_{l, m} l \hat{P}_{u^{l-1}, w^{m}} a_{l, m, x} \circ\left(a_{x}^{\otimes(l-1)} \otimes E_{x}^{\otimes m}\right) a_{x} d t \\
& +\sum_{l, m} m \hat{P}_{u^{l}, w^{m-1}} a_{l, m, x} \circ\left(a_{x}^{\otimes l} \otimes E_{x}^{\otimes(m-1)}\right) E_{x} d w_{x} .
\end{aligned}
$$


Hence

$$
f\left(\xi_{x}^{0}(t, \omega)\right)=\left.\sum_{l, m} \hat{P}_{u^{l}, w} m a_{l, m, f(x)}^{f}\left(u, f\left(\xi_{x}^{0}(u, \omega)\right)\right) \circ\left(a_{f(x)}^{f} \otimes l \otimes E_{f(x)}^{f}{ }^{\otimes m}\right)\right|_{u=t} .
$$

Therefore, $f^{*}: \exp _{x}^{M *}\left(d \xi_{x}^{0}\right) \mapsto \exp _{f(x)}^{N *}\left(f^{*} d \xi_{x}^{0}\right)$, where

$$
\begin{aligned}
& f^{*} d \xi_{x}^{0}=\sum_{l, m} l \hat{P}_{u^{l-1}, w^{m}} a_{l, m, f(x)}^{f} \circ\left(a_{f(x)}^{f}{ }^{\otimes(l-1)} \otimes E_{f(x)}^{f}{ }^{\otimes m}\right) a_{f(x)}^{f} d t
\end{aligned}
$$

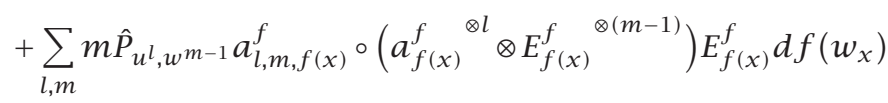

for $f$-related mappings $\exp ^{M}$ and $\exp ^{N}$.

THEOREM 2.13. Let $\exp : \tilde{T} M \rightarrow M$ be the exponential mapping of a manifold $M$. Then $J(\exp , a, E): J^{\tilde{T} M} \rightarrow J^{M}$ is a bundle morphism. If $(U, \phi)$ is a chart of $M$, then

$$
J\left(\exp _{\phi(x)}^{\phi}, a_{x}^{\phi}, E_{x}^{\phi}\right)\left(a_{x}^{\phi}, E_{x}^{\phi}\right)=\left(S a_{x}^{\phi}, S E_{x}^{\phi}\right),
$$

where $S:=\left.\left(d\left[\phi \circ \exp _{x} \circ\left[\phi^{\prime}(x)\right]^{-1}\right](z) / d z\right)\right|_{z=\xi_{x}^{0}}$.

Proof. The first statement of the theorem follows from Theorem 2.11 and Corollary 2.12. Consider the mapping $F(z):=\left[\phi \circ \exp _{x} \circ\left[\phi^{\prime}(x)\right]^{-1}\right](z)$ for a chart $\left(T U_{j}, T \phi_{j}\right)$ of $T M$, where $\phi=\phi_{j}$. The mapping $F$ is the local representation of exp in terms of coordinate mappings. Hence $J\left(\exp _{\phi(x)}^{\phi}, a_{x}^{\phi}, E_{x}^{\phi}\right)=\left.[d F(z) / d z]\right|_{z=\xi_{x}^{0}}$, where $\xi_{x}^{0}$ is a solution of either (2.13) or (2.16) in $T_{x} M$. In particular, $F^{\prime}(0)=$ id and $F^{\prime \prime}(0) \cdot(v, v)=-\Gamma(x)(v, v)$, but in general $\xi_{x}^{0}$ may be nonzero.

Definition 2.14. Let $U$ be a section of the bundle $(\Pi, M, \pi)$. Consider the differential

$$
d \xi(t, \omega)=\exp _{\xi(t, \omega)}^{*} \mathscr{G}\left(a_{\left.\xi(t, \omega), E_{\xi(t, \omega)}\right)}\right.
$$

and the corresponding antiderivational equation

$$
\begin{array}{r}
\xi(t, \omega)=\exp _{\xi(t, \omega)}\left\{\sum_{l, b, m} \hat{P}_{u^{l+b}, w} m a_{b+l, m, \xi(t, \omega)}(u, \xi(u, \omega))\right. \\
\left.\left.\circ\left(I^{\otimes b} \otimes a_{\xi(t, \omega)}^{\otimes l} \otimes E_{\xi(t, \omega)}^{\otimes m}\right)\right|_{u=t}\right\} .
\end{array}
$$

Suppose that there exist a neighborhood $V_{x} \ni x$ and a stochastic process belonging to

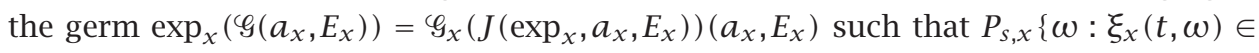

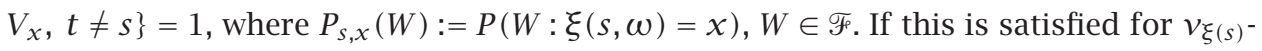
a.e. $x \in M$, then it is said that $\xi(t, \omega)$ possesses a stochastic differential governed by the field $U$, where $v_{\xi(s)}(*):=P \circ \xi^{-1}(s, *)$. An $M$-valued $\xi$ satisfying (2.32) is called an integral process of the field $u(t)$. 
Definition 2.15. An atlas $\operatorname{At}(M)=\left\{\left(U_{j}, \phi_{j}\right): j\right\}$ of a manifold $M$ on a Banach space $X$ over $\mathbf{K}$ is called uniform if its charts satisfy the following conditions:

(U1) for each $x \in M$, there exist neighborhoods $U_{x}^{2} \subset U_{x}^{1} \subset U_{j}$ such that for each $y \in U_{x}^{2}$, there is the inclusion $U_{x}^{2} \subset U_{y}^{1}$;

(U2) the image $\phi_{j}\left(U_{x}^{2}\right) \subset X$ contains a ball of the fixed positive radius $\phi_{j}\left(U_{x}^{2}\right) \supset$ $B(X, 0, r):=\{y: y \in X,\|y\| \leq r\}$

(U3) for each pair of intersecting charts $\left(U_{1}, \phi_{1}\right)$ and $\left(U_{2}, \phi_{2}\right)$, transition mappings $\phi_{l, j}=\phi_{l} \circ \phi_{j}^{-1}$ are such that $\sup _{x}\left\|\phi_{l, j}^{\prime}\right\| \leq C$ and $\sup _{x}\left\|\phi_{l, j}(x)\right\| \leq C$, where $C=$ const $>0$ does not depend on $\phi_{l}$ and $\phi_{j}$.

REMARK 2.16. Consider a measurable space $(M, \mathscr{L})$, where $\mathscr{L}$ is a $\sigma$-algebra on $M$. Define a random mapping $S(t, \tau ; \omega): M \rightarrow M$ for each $t, \tau \in T$ by $x \mapsto S(t, \tau ; \omega ; x)=$ $S(t, \tau ; \omega) \circ x$. Suppose that

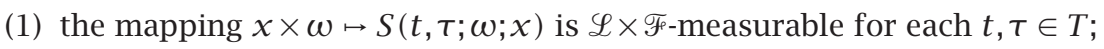

(2) the random variable $S(t, \tau ; \omega ; x)$ is $\mathscr{F}$-measurable and does not depend on $\mathscr{F}$ for each $t, \tau, x$. Moreover, let also all other conditions of [30, Definition 3.8] be satisfied (with the notation $S(t, \tau ; \omega)$ here instead of $T(t, s ; \omega)$ there).

Proposition 2.17. Let $\xi$ be a stochastic process given by (2.16) and let also max $(\| a(t$, $\omega, x)-a(v, \omega, x)\|\| E,(t, \omega, x)-E(v, \omega, x) \|) \leq|t-v|\left(C_{1}+C_{2}\|x\|^{b}\right)$ for each $t$ and $v \in B\left(\mathbf{K}, t_{0}, R\right) \lambda$-almost everywhere by $\omega \in \Omega$, where $b, C_{1}$, and $C_{2}$ are nonnegative constants. Then $\xi$ with probability 1 has a $C^{0}$-modification and $q(t) \leq \max \left\{M\left\|\xi_{0}\right\|^{s}, \mid t-\right.$ $\left.t_{0} \mid\left(C_{1}+C_{2} q(t)\right)\right\}$ for each $t \in B\left(\mathbf{K}, t_{0}, R\right)$, where $q(t):=\sup _{\left|u-t_{0}\right| \leq\left|t-t_{0}\right|} M\|\xi(u, \omega)\|^{s}$ and $\mathbb{N} \ni s \geq b \geq 0$. Moreover, if $\lambda\left\{\omega: w(t, \omega) \in C^{0} \backslash C^{1}\right\}=0$, then for $\lambda$-a.e. $\omega$, there exists $\xi^{\prime}$ and $\lambda\left\{\omega: \xi(t, \omega) \in C^{0} \backslash C^{1}\right\}=0$.

Proof. In view of Theorem 2.8 applied to $f(t, x)=x^{s}$, we have

$$
\begin{aligned}
f(t, \xi(t, \omega))= & f\left(t_{0}, \xi_{0}\right) \\
+\sum_{k=1}^{s} \sum_{l_{1}, \ldots, l_{k}}\left(\hat { P } _ { u ^ { k - l } , w ( u , \omega ) ^ { l } } \left[\left(\begin{array}{l}
s \\
k
\end{array}\right) \xi^{s-k}(u, \xi(u, \omega))\right.\right. & \left.\left.\circ\left(a_{l_{1}, n_{1}} \otimes \cdots \otimes a_{l_{k}, n_{k}}\right) \circ\left(a^{\otimes(k-l)} \otimes E^{\otimes l}\right)\right]\right)\left.\right|_{u=t},
\end{aligned}
$$

where $l_{1}+\cdots+l_{k}=k-l, n_{1}+\cdots+n_{k}=l$. From the conditions of Note 2.7, and in particular (2.17), it follows that

$$
M\|\xi(t, \omega)\|^{s} \leq \max \left(M\left\|\xi_{0}\right\|^{s},\left|t-t_{0}\right| d\left(\hat{P}_{*}^{s}\right)\left(C_{1}+C_{2} \sup _{\left|u-t_{0}\right| \leq\left|t-t_{0}\right|} M\|\xi(u, \omega)\|^{s}\right)\right)
$$

since $\left|t_{j}-t_{0}\right| \leq\left|t-t_{0}\right|$ for each $j \in \mathbb{N}$, and $M\|\xi(t, \omega)-\xi(v, \omega)\|^{s} \leq|t-v|\left(1+C_{1}+\right.$ $\left.C_{2} d\left(\hat{P}_{*}^{s}\right) \sup _{\left|u-t_{0}\right| \leq \max \left(\left|t-t_{0}\right|,\left|v-t_{0}\right|\right)} M\|\xi(u, \omega)\|^{s}\right)$ since $\left|t_{j}-v_{j}\right| \leq|t-v|+\rho^{j}$ for each 
$j \in \mathbb{N}$, where $0<\rho<1$,

$$
\begin{aligned}
& d\left(\hat{P}_{*}^{s}\right) \\
& :=\operatorname{mup}_{\left(a \neq 0, E \neq 0, f \neq 0, a_{l_{j}, n_{j}} \neq 0, j=1, \ldots, k\right)} \max _{s \geq k \geq l \geq 0} \|(k !)^{-1} \hat{P}_{u^{k-l}, w^{l}}\left(\frac{\partial^{k} f}{\partial^{k} x}\right) \\
& \quad \times \frac{1}{\left(\|a\|_{C^{0}\left(B_{R}, H\right)}^{k-l}\|E\|_{C^{0}\left(B_{R}, L(H)\right)}^{l}\|f\|_{C^{s}\left(B_{R}, H\right)} \prod_{j=1}^{k}\left\|a_{l_{j}, n_{j}}\right\|\right)},
\end{aligned}
$$

hence $d\left(\hat{P}_{*}^{s}\right) \leq 1$, since $f \in C^{s}$ as a function by $x$ and $\left(\bar{\Phi}^{s} g\right)\left(x ; h_{1}, \ldots, h_{s} ; 0, \ldots, 0\right)=$ $D_{x}^{s} g(x) \cdot\left(h_{1}, \ldots, h_{s}\right) / s$ ! for each $g \in C^{s}$ and due to the definition of $\|g\|_{C^{s}}$. Considering in particular polyhomogeneous $g$ on which $d\left(\hat{P}_{*}^{s}\right)$ takes its maximum value, we get $d\left(\hat{P}_{*}^{s}\right)=1$. From conditions on $w, a_{l, k}, a$, and $E$, it follows that $\xi(t, \omega)$ with probability 1 has a $C^{0}$-modification (see [30, Theorem 3.4]), $\xi \in L^{q}\left(\Omega, \mathscr{F}, \lambda ; C^{0}\left(B_{R}, H\right)\right)$.

The last statement of this proposition follows from [31, Lemma 2.2].

THEOREM 2.18. Suppose that $M$ is a manifold which either satisfies the conditions of Corollary 2.5 or is analytic, $\operatorname{At}(M)$ is uniform (see Definition 2.15). Let $a, E, a_{m, l}$, and $w$ corresponding to a section $U$ satisfy the conditions of Note 2.7 with $\lambda\{\omega: w(t, \omega) \in$ $\left.C^{0} \backslash C^{1}\right\}=0$. Then there exists a unique up to stochastic equivalence random evolution family $S(t, \tau ; \omega)$ for a solution $\xi(t, \omega)$ of $(2.32)$.

Proof. Consider a solution of the non-Archimedean stochastic equation

$$
\begin{array}{r}
\xi(t, \omega)=\exp _{\xi(t, \omega)}\left\{\sum_{m, b, l} \hat{P}_{u^{m+b}, w^{l}} a_{m+b, l, \xi(t, \omega)}(u, \xi(u, \omega))\right. \\
\left.\left.\circ\left(I^{\otimes b} \otimes a_{\xi(t, \omega)}^{\otimes m} \otimes E_{\xi(t, \omega)}^{\otimes l}\right)\right|_{u=t}\right\}
\end{array}
$$

corresponding to (2.31). On each chart of the uniform atlas $\operatorname{At}(M)$ of $M$ fields $\left\{a_{m, l}\right.$ : $m, l\}, a, E$, and $w$ are $\lambda$-a.e. bounded due to the conditions of Note 2.7. For each two charts $\left(U_{j}, \phi_{j}\right)$ and $\left(U_{l}, \phi_{l}\right)$ with $U_{j} \cap U_{l} \neq \varnothing$, a transition mapping $\phi:=\phi_{j, l}$ is bounded together with its derivatives, hence $\Gamma$ is bounded on each $U_{j}$ since the covering $\left\{U_{j}: j\right\}$ of $M$ can be chosen locally finite due to paracompactness of $M$ [6].

In view of [30, Theorem 3.4], Corollary 2.12 and equation (2.30) have a unique solution on $M$. Let $(a, E)$ be a section of the bundle $(\Pi, M, \pi)$ and $a_{l, m}$ sections of the bundles $\left(\Pi_{l+m}, M, \pi_{l+m}\right)$ (see Definition 2.10 and Section 2.5). Consider a family $\zeta_{y}(x)$ of functions of the class $C^{1}(M, \mathbf{K})$ on $M$ such that $\zeta_{y}(x)=0$ if $x \notin U_{y}^{1}, \zeta_{y}(x)=1$ if $x \in U_{y}^{2}$ of the uniform atlas (see Definition 2.15), then $a_{x}^{y}:=\zeta_{y}(x) a_{x}, E_{x}^{y}:=\zeta_{y}(x) E_{x}, a_{l, m, x}^{y}:=$ $\zeta_{y}(x) a_{l, m}$ are local fields. Then, there exists the local evolution family $S_{y}(t, \tau ; \omega)$ for 
each local solution (i.e, with local coefficients)

$$
\begin{array}{r}
\xi^{y}(t, \omega)=\exp _{\xi^{y}(t, \omega)}\left\{\sum_{m, b, l} \hat{P}_{u^{m+b}, w^{l}} a_{m+b, l, \xi^{y}(t, \omega)}^{y}(u, \xi(u, \omega))\right. \\
\quad \circ\left(\left.I^{\otimes b} \otimes\left(a_{\xi^{y}(t, \omega)}^{y}\right)^{\otimes m} \otimes\left(E_{\xi^{y}(t, \omega)}^{y}\right)\right|_{u=t}\right\},
\end{array}
$$

due to [30, Theorem 3.4] and Theorem 2.8 above. Therefore, $S_{y}(t, \tau ; \omega) \circ x \in U_{y}^{1}$ for each $x \in U_{y}^{2}$. Glueing together local solutions with the help of transition functions $\phi_{l, j}$ of charts with nonvoid intersections $U_{l} \cap U_{j}$ leads to the conclusion that a stochastic process $\xi$ is a solution of the stochastic antiderivational equation (2.36) on $M$, if and only if, for each $t \in T$ for $\nu_{\xi(t)}$-a.e. $x \in M$, it coincides $P_{t, x}$-a.e. with some local solution of this equation inside $U_{x}^{2}$, since $\left\{U_{x}^{2}: x \in M\right\}$ is a covering of $M$.

Consider a local representation $\xi^{\phi}:=\phi(\xi)$, then there exists the corresponding $S^{\phi}$ generated by $d \xi^{\phi}$ such that $S^{\phi}(t, \tau ; \omega) \circ \xi^{\phi}(\tau, \omega)=\phi(S(t, \tau ; \omega) \circ \xi(\tau, \omega))$ for each $t$, $\tau \in T \subset \mathbf{K}, \phi \in\left\{\phi_{j}: j\right\}$.

In view of Proposition 2.17, there exists $\delta>0$ such that $P\left\{\omega: S_{x}(t, \tau ; \omega) \circ x \notin U_{x}^{2}\right\} \leq$ $P\left\{\sup \left\|\phi\left(S_{x}(t, \tau ; \omega) \circ x\right)\right\|>1\right\} \leq C|t-\tau|$ for each $t, \tau \in T$, such that $|t-\tau|<\delta$, where $C>0$ is a constant. Consider a family $Y$ of all finite partitions $q$ of $T$ into disjoint unions of balls $B\left(\mathbf{K}, t_{k}^{q}, r_{k}^{q}\right)$, where $t_{k}^{q} \in T, 0<r_{k}^{q} \leq \epsilon_{q}, 0<\epsilon_{q}<\delta$ for each $q \in \Upsilon$. Let $q \leq v$ if and only if $q \subset v$, then $\mathrm{Y}$ is ordered by this relation. Consider a linearly ordered subsequence $\Upsilon_{0}:=\left\{q_{k}: k \in \mathbb{N}\right\}$ in $\Upsilon$ with $\lim _{k \rightarrow \infty} \sup \left\{r_{j}^{q_{k}}: j \in q_{k}\right\}=0$, and for it, define $\xi_{k}(t, \omega):=S_{\xi_{k-1}\left(t_{l}, \omega\right)}\left(t, t_{l} ; \omega\right) \circ \xi_{k-1}\left(t_{l}, \omega\right)$ for each $k$ and $t \in B\left(\mathbf{K}, t_{l}^{v}, r_{l}^{v}\right)$ for each $t_{l} \in q_{k-1}$ and each $k \geq 1$, where $v=q_{k-1}, \xi\left(t_{0}, \omega\right)=x, \xi_{1}\left(t_{l}, \omega\right):=\xi\left(t_{l}, \omega\right)$ for each $t_{l} \in q_{1}$. Also define $S^{k}\left(t, t_{0} ; \omega\right) \circ x=S_{\xi_{k-1}\left(t_{l}, \omega\right)}\left(t, t_{l} ; \omega\right) \circ \xi_{k-1}\left(t_{l}\right)$. Consider $z(s, \omega):=$ $S_{y}\left(s, t_{k}^{q} ; \omega\right) \circ y \in U_{y}^{2}$ for each $s \in B\left(\mathbf{K}, t_{k}^{q}, r_{k}^{q}\right)$. For each $t \in B\left(\mathbf{K}, t_{k}^{q}, r_{k}^{q}\right)$, the equality $S_{y}\left(t, t_{k}^{q} ; \omega\right) \circ y=S_{z(s, \omega)}(t, s ; \omega) \circ z(s, \omega)$ is satisfied since $S_{y}\left(t, t_{k}^{q} ; \omega\right) \circ y=S_{y}(t, s ; \omega) \circ$ $S_{y}\left(s, t_{k}^{q} ; \omega\right) \circ y$ due to the existence of a local solution.

Put $\Omega^{\Upsilon_{0}}:=\bigcup_{k \in Y_{0}} \Omega_{k}$, where $\Omega_{k}:=\bigcap_{l \in q_{k}} \Omega_{k, l}$, where $\Omega_{k, l}:=\left\{\omega: S_{\xi_{k}\left(t_{l}, \omega\right)}\left(s, t_{l}\right) \circ \xi_{k}\left(t_{l}, \omega\right)\right.$ $\left.\in U_{\xi_{k}\left(t_{l}, \omega\right)}^{2}, s \in B\left(\mathbf{K}, t_{l}^{v}, r_{l}^{v}\right)\right\}$. From the existence of a local solution, it follows that $S^{k}\left(t, t_{0}\right) \circ x=S^{l}\left(t, t_{0}\right) \circ x$ for each $k \geq l$ and each $\omega \in \Omega_{l}$. In view of Theorem 2.13, there exists $\lim _{q_{k} \in Y_{0}} S^{k}\left(t, t_{0} ; \omega\right)=S\left(t, t_{0} ; \omega\right)$. For each two linearly ordered subsets $\Lambda_{1}$ and $\Lambda_{2}$ in $\Upsilon$, there exists a linearly ordered subset $\Lambda$ in $\Upsilon$ such that $\Lambda \supset \Lambda_{1} \cup \Lambda_{2}$, hence this limit does not depend on the choice of $\Upsilon_{0}$. Events $\Omega_{k, l}$ and $\Omega_{k, j}$ are independent in total for each $l \neq j: P\left(\Omega_{k, l} \cap \Omega_{k, j}\right)=P\left(\Omega_{k, l}\right) P\left(\Omega_{k, j}\right)$. Since $\mathbf{K}$ is a finite algebraic extension of the corresponding $\mathbf{Q}_{p}$, then there exists $n \in \mathbb{N}$ such that $\mathbf{K}$ as the $\mathbf{Q}_{p}$ linear space is isomorphic to $\mathbf{Q}_{p}^{n}$. Choose $\Upsilon_{0}$ such that for each $q_{k} \in \Upsilon_{0}$, the supremum $\sup _{l \in q_{k}} r_{l}^{q_{k}}=: \delta_{k} \leq p^{-k}$ and $\operatorname{card}\left(t_{l}: t_{l} \in q_{k} \cap B\left(\mathbf{K}, t_{0}, p^{s}\right)\right)=: m_{k, s} \leq p^{s n k}$. In view of the ultrametric inequality from $\|\alpha(\omega)+\beta(\omega)\| \geq \delta$, it follows that $\max (|\alpha(\omega)|,|\beta(\omega)|) \geq \delta$ for each two random variables $\alpha$ and $\beta$. Therefore, from Proposition 2.17 applied to $\phi_{j}(\xi)-\phi_{j}\left(\xi_{0}\right)$, and the inclusion $\xi(t, \omega) \in L^{q}\left(\Omega, \mathscr{F}, \lambda ; C^{0}(T, M)\right)$, it follows that $P\left\{\Omega_{k}\right.$ : $\left.t \in T \cap B\left(\mathbf{K}, t_{0}, p^{s}\right)\right\} \geq\left(1-C_{k} p^{-k}\right)^{p^{s n k}}$, where $\lim _{k} C_{k}=0$, since $\xi(t, \omega)$ is uniformly continuous on $T \cap B\left(\mathbf{K}, t_{0}, p^{s}\right)$ for $\lambda$-a.e. $\omega$. Therefore, $P\left\{\Omega^{\Upsilon_{0}}: t \in T \cap B\left(\mathbf{K}, t_{0}, p^{s}\right)\right\} \geq$ $\lim _{k} \exp \left(-C_{k} s n\right)=1$ for each given $s \in \mathbb{N}$. 
From $S^{k}\left(t, t_{0} ; \omega\right) \circ x=S^{k}(t, s ; \omega) \circ S^{k}\left(s, t_{0} ; \omega\right) \circ x$ and taking the limit by $q \in Y$, it follows that $S$ satisfies the evolution property $S\left(t, t_{0} ; \omega\right) \circ x=S(t, s ; \omega) \circ S\left(s, t_{0} ; \omega\right) \circ x$. Then $S\left(t, t_{0} ; \omega\right) \circ x$ is a measurable function of $x$, since it is a superposition $S\left(t, t_{0} ; \omega\right) \circ$ $x=S^{k}\left(t, t_{0} ; \omega\right) \circ x$ of locally measurable functions.

COROLLARY 2.19. Let the conditions of Theorem 2.18 be satisfied and let $f$ be a function on $T \times M$ such that each $f \circ \phi_{j}^{-1}$ satisfies conditions (2.18) and (2.19) on its domain. Then, a generating operator of an evolution family $S(t, \tau ; \omega)$ of a stochastic process $\eta(t, \omega)=f(t, \xi(t, \omega))$ is given by the equation

$$
\begin{aligned}
& A(t ; \omega) \eta(t, \omega)= \sum_{\substack{m+b \geq 1,0 \leq m \in \mathbb{Z} \\
0 \leq b \in \mathbb{Z}}}((m+b) !)^{-1} \sum_{l=0}^{m}\left(\begin{array}{c}
m+b \\
m
\end{array}\right) \\
& \times \sum_{\substack{l_{1}+\cdots+l_{m}=m-l \\
n_{1}+\cdots+n_{m}=l}}\left\{b \left(\hat { P } _ { u ^ { b + m - l - 1 } , w ( u , \omega ) ^ { l } } \left[\left(\frac{\partial^{b} \nabla^{m} f}{\partial u^{b} \partial x^{m}}\right)(u, \xi(u, \omega))\right.\right.\right. \\
& \circ\left(a_{l_{1}, n_{1}} \otimes \cdots \otimes a_{l_{m}, n_{m}}\right) \\
&+(m-l)\left(\hat { P } _ { u ^ { b + m - l - 1 } , w ( u , \omega ) ^ { l } } \left[\left(\frac{\partial^{b} \nabla^{m} f}{\partial u^{b} \partial x^{m}}\right)(u, \xi(u, \omega))\right.\right. \\
& \circ\left(a_{l_{1}, n_{1}} \otimes \cdots \otimes a_{l_{m}, n_{m}}\right)\left.\left.\left.\left.\left.\circ\left(I^{\otimes b} \otimes a^{\otimes(m-l-1)} \otimes E^{\otimes l}\right)\right] a\right)\left.\right|_{u=t} ^{\otimes(m-l)} \otimes E^{\otimes l}\right)\right]\right)\left.\right|_{u=t} \\
&+l\left(\hat { P } _ { u ^ { b + m - l } , w ( u , \omega ) ^ { l - 1 } } \left[\left(\frac{\partial^{b} \nabla^{m} f}{\partial u^{b} \partial x^{m}}\right)(u, \xi(u, \omega))\right.\right. \\
& \circ\left(a_{l_{1}, n_{1}} \otimes \cdots \otimes a_{l_{m}, n_{m}}\right) \\
&\left.\left.\left.\circ\left(I^{\otimes b} \otimes a^{\otimes(m-l)} \otimes E^{\otimes(l-1)}\right)\right] E w^{\prime} u(u, \omega)\right)\left.\right|_{u=t}\right\} .
\end{aligned}
$$

Proof. In view of Theorem 2.18, there exists a generating operator $S(t, \tau ; \omega)$ of an evolution family. For each chart $\left(U_{j}, \phi_{j}\right)$, the stochastic process $f \circ \phi_{j}^{-1}(\xi)$ is given by (2.20). Consider the covariant differentiation $(\nabla f / \partial x) \cdot h=\nabla_{h} f$ on the manifold $M$, where $h \in T_{X} M$. For a random variable belonging to $L^{q}\left(\Omega, \mathscr{F}, \lambda ; C^{1}(M, X)\right)$, its derivative and partial difference quotients $\bar{\Phi}^{1} f \circ \phi_{j}^{-1}(x ; h ; b)$ are naturally understood as elements of the corresponding spaces $L^{q}\left(\Omega, \mathscr{F}, \lambda ; C^{0}\left(W_{j}, X\right)\right)$ such that each limit $\lim _{x \rightarrow x_{0}} g(x, \omega)=c(\omega)$ is taken in $L^{q}\left(\Omega, \mathscr{F}, \lambda ; C^{0}(M, X)\right)$, where $W_{j}:=\left\{(x, h, b) \in U_{j} \times\right.$ $\left.X \times \mathbf{K}: x+b h \in U_{j}\right\}$. In other words, it exists if and only if $\lim _{x \rightarrow x_{0}}\|g(x, \omega)-c(\omega)\|_{L^{q}}=$ 0 , where $c \in L^{q}(\Omega, \mathscr{F}, \lambda ; X)$. Then $f(t, \xi(t, \omega))=\lim _{k} f\left(t, S^{k}\left(t, t_{0} ; \omega\right) \circ x\right)$. For each chart, put $f_{j}(t, *):=f\left(t, \phi_{j}^{-1}(*) \phi_{j} \circ S_{y}\right)$, where $S_{y}(t, \tau ; \omega) y$ does not leave for $\lambda$-a.e. $\omega \in \Omega$ a clopen subset $U_{j}$ in $M$ for each $t$ and $\tau \in T_{j}, T_{j} \subset T, \cup_{j} T_{j}=T, T_{j}$ is a clopen subset in 
$T$. Then define $f\left(t, S^{k}(t, \tau ; \omega) x\right)_{x}^{\prime} \cdot h=f_{x}^{\prime}\left(t, S^{k}(t, \tau) \circ x\right) A^{k}(t, \tau ; \omega) h$ and take the limit. From [31, Lemma 2.2], the statement of this corollary follows.

REMARK 2.20. In the particular case in Section 2.4, formula (2.38) simplifies. When the family of $\Gamma$, together with all its covariant derivatives along $a$ and $E w^{\prime}$, is equiuniformly bounded on each $U_{j}$, then (2.38) can be written in another form using the identity $\nabla^{m+1} f \cdot\left(h_{1}, \ldots, h_{m}\right)=\nabla_{h_{m+1}}\left(\nabla^{m} f \cdot\left(h_{1}, \ldots, h_{m}\right)\right)-\sum_{l=1}^{m}\left(\nabla^{m} f\right) \cdot\left(h_{1}, \ldots, h_{l-1}, \nabla_{h_{m+1}} h_{l}\right.$, $\left.h_{l+1}, \ldots, h_{m}\right)$, in particular with $h_{l} \in\left\{a, E w^{\prime}\right\}$.

In a weak topology, $C^{0}\left(T, c_{0}(\alpha, \mathbf{K})\right)$ is isomorphic to $c_{0}(\alpha, \mathbf{K})^{T}$. Let $\theta: \mathbf{K} \rightarrow \mathbb{R}$ be a continuous surjective quotient mapping, such that $\theta(B(\mathbf{K}, 0,1))=[0,1]$. Then, for each $\xi \in L^{q}\left(\Omega, \mathscr{F}, \lambda ; c_{0}(\alpha, \mathbf{K})^{T}\right)$, there exists $\theta(\xi) \in L^{q}\left(\Omega, \mathscr{F}, \lambda ; c_{0}(\alpha, \mathbb{R})^{\theta(T)}\right)$ that induces a surjective mapping $\theta^{*}$ from $L^{q}\left(\Omega, \mathscr{F}, \lambda ; c_{0}(\alpha, \mathbf{K})^{T}\right)$ onto $L^{q}\left(\Omega, \mathscr{F}, \lambda ; c_{0}(\alpha, \mathbb{R})^{\theta(T)}\right)$. Therefore, for each stochastic process $\eta$ in $L^{q}\left(\Omega, \mathscr{F}, \lambda ; c_{0}(\alpha, \mathbb{R})^{\theta(T)}\right)$, there exists a stochastic process $\xi$ in $L^{q}\left(\Omega, \mathscr{F}_{F}, \lambda ; \mathcal{c}_{0}(\alpha, \mathbf{K})^{T}\right)$ such that $\theta(\xi)=\eta$. On the other hand, $\mathbf{K}$ is a projective limit of discrete rings $S_{\pi^{n}}$ isomorphic to the quotient of $\mathbf{K}$ by the equivalence relation associated with disjoint subsets $x_{j}+\pi^{n} B(\mathbf{K}, 0,1)$ in $\mathbf{K}, j=0,1,2, \ldots, x_{0}:=0, \pi \in \mathbf{K}$, $|\pi|=\max \{|x|:|x|<1, x \in \mathbf{K}\}$. Therefore, $L^{q}\left(\Omega, \mathscr{F}_{,}, \lambda ; \mathcal{C}_{0}(\alpha, \mathbf{K})^{T}\right)$ is isomorphic as the topological space to projective limit of modules $L^{q}\left(\Omega, \mathscr{F}, \lambda ; \mathcal{C}_{0}\left(\alpha, \mathbf{S}_{\pi^{n}}\right)^{\mathbf{S}_{\pi^{n}}}\right)$ over discrete rings $\mathbf{S}_{\pi^{n}}$. Since simple functions are dense in $L^{q}$, consequently, $\xi$ is equal to the projective limit of stochastic processes with values in discrete modules $c_{0}\left(\alpha, \mathbf{S}_{\pi^{n}}\right)$ over rings $\mathbf{S}_{\pi^{n}}$. This opens a possibility of approximation of stochastic processes by stochastic processes with values in discrete modules. Certainly, there is not any simple relation between classical and non-Archimedean stochastic equations, so, if $\xi$ satisfies definite stochastic antiderivational equation relative to $w$, it is a problem to find a classical stochastic equation which $\theta(\xi)$ satisfies relative to either $\theta(w)$ or a standard stochastic process (Wiener, Lèvy) and vice versa.

Theorem 2.18 and Corollary 2.19 are applicable in particular to totally disconnected Lie groups over non-Archimedean fields of characteristic zero.

In [29], wide classes of nonnegative (in particular also quasi-invariant) measures $v$ on non-Archimedean Banach spaces $Y$ were considered, as well as having abundant families of compact subsets of positive measure. Then $v$ induces a (quasi-invariant) measure $\mu$ on a manifold $M$ modelled on $Y$ (see [21, Theorem 3.2], which can be generalized to the class of manifolds considered here). If $f \in L^{1}(M, \mu, \mathbb{C})$, then $\lambda(d x)=f(x) \mu(d x)$ is a measure on $M$. In particular, $f$, and hence $\lambda$ with a compact support, can be considered, but with losing its quasi-invariance property. Therefore, stochastic processes having transition measures of compact supports are particular cases of those considered herein.

ACKNOWLedgment. The author thanks Professor J. Oesterle and Professor H. Rosenberg for hospitality at the "Institut de Mathématiques," Université Paris 6 et 7, and the "Minister of the Research" of France for support.

\section{REFERENCES}

[1] S. Albeverio and X. Zhao, On the relation between different constructions of random walks on $p$-adics, Markov Process. Related Fields 6 (2000), no. 2, 239-255. 
[2] Ya. I. Belopol'skaya and Yu. L. Dalecky, Stochastic Equations and Differential Geometry, Mathematics and Its Applications (Soviet Series), vol. 30, Kluwer Academic Publishers, Dordrecht, 1990.

[3] A. Kh. Bikulov and I. V. Volovich, p-adic Brownian motion, Izv. Ross. Akad. Nauk Ser. Mat. 61 (1997), no. 3, 75-90.

[4] D. R. Cox and H. D. Miller, The Theory of Stochastic Processes, John Wiley \& Sons, New York, 1965.

[5] Yu. L. Dalecky and S. V. Fomin, Measures and Differential Equations in Infinite-Dimensional Space, Mathematics and Its Applications (Soviet Series), vol. 76, Kluwer Academic Publishers, Dordrecht, 1991.

[6] R. Engelking, General Topology, Mir, Moscow, 1986.

[7] S. N. Evans, Continuity properties of Gaussian stochastic processes indexed by a local field, Proc. London Math. Soc. (3) 56 (1988), no. 2, 380-416.

[8]__ Local field Brownian motion, J. Theoret. Probab. 6 (1993), no. 4, 817-850.

[9] __ $p$-adic white noise, chaos expansions, and stochastic integration, Probability Measures on Groups and Related Structures XI (Oberwolfach, 1994), World Sci. Publishing, New Jersey, 1995, pp. 102-115.

[10] _ Local fields, Gaussian measures, and Brownian motions, Topics in Probability and Lie Groups: Boundary Theory, CRM Proc. Lecture Notes, vol. 28, American Mathematical Society, Rhode Island, 2001, pp. 11-50.

[11] J. Fresnel and M. van der Put, Rigid Analytic Geometry and Its Applications, Progress in Mathematics, vol. 18, Birkhäuser Boston, Massachusetts, 1981.

[12] I. I. Gīhman and A. V. Skorohod, Stochastic Differential Equations, Izdat. "Naukova Dumka”, Kiev, 1968.

[13] _ The Theory of Stochastic Processes. II, Springer-Verlag, New York, 1975.

[14] K. Itô and H. P. McKean Jr., Diffusion Processes and Their Sample Paths, Springer-Verlag, Berlin, 1974.

[15] Y. Jang, Non-Archimedean Quantum Mechanics, Tohoku Mathematical Publications, vol. 10, Tohoku University Mathematical Institute, Sendai, 1998.

[16] A. Khrennikov, Interpretations of Probability, VSP, Utrecht, 1999.

[17] A. Yu. Khrennikov, Generalized functions and Gaussian path integrals over nonArchimedean function spaces, Izv. Akad. Nauk SSSR Ser. Mat. 55 (1991), no. 4, 780814.

[18] W. Klingenberg, Riemannian Geometry, de Gruyter Studies in Mathematics, vol. 1, Walter de Gruyter, Berlin, 1982.

[19] A. N. Kochubei, Pseudo-Differential Equations and Stochastics over Non-Archimedean Fields, Monographs and Textbooks in Pure and Applied Mathematics, vol. 244, Marcel Dekker, New York, 2001.

[20] S. V. Ludkovsky, Non-Archimedean stochastic processes on non-Archimedean manifolds, preprint, 1999, http://arxiv.org/abs/math.GM/0212296.

[21]__ Irreducible unitary representations of non-Archimedean groups of diffeomorphisms, Southeast Asian Bull. Math. 22 (1998), no. 4, 419-436.

[22] _ Quasi-invariant measures on non-Archimedean semigroups of loops, Russ. Math. Surveys 53 (1998), no. 3, 633-634.

[23] _ Measures on diffeomorphism groups of non-Archimedean manifolds, representations of groups and their applications, Theoret. and Mathem. Phys. 119 (1999), no. 3, 698-711. , Properties of quasi-invariant measures on topological groups and associated algebras, Ann. Math. Blaise Pascal 6 (1999), no. 1, 33-45. , Quasi-invariant measures on non-Archimedean groups and semigroups of loops and paths, their representations. I, Ann. Math. Blaise Pascal 7 (2000), no. 2, 19-53. , Quasi-invariant measures on non-Archimedean groups and semigroups of loops and paths, their representations. II, Ann. Math. Blaise Pascal 7 (2000), no. 2, 55-80. 
[27]

[29]

[30]

[31]

[32] , Stochastic processes on groups of diffeomorphisms and loops of real, complex, and non-Archimedean manifolds, Fundam. Prikl. Mat. 7 (2001), no. 4, 1091-1105 (Russian).

, Poisson measures for topological groups and their representations, Southeast Asian Bull. Math. 25 (2002), no. 4, 653-680.

, Quasi-invariant and pseudo-differentiable measures on a non-Archimedean Banach spaces, Anal. Math. 28 (2002), no. 4, 287-316 (Russian).

, Stochastic antiderivation equations on non-Archimedean Banach spaces, Int. J. Math. Math. Sci. 2003 (2003), no. 41, 2587-2602.

, Stochastic processes on non-Archimedean Banach spaces, Int. J. Math. Math. Sci. 2003 (2003), no. 21, 1341-1363.

, Stochastic processes on totally disconnected topological groups, Int. J. Math. Math. Sci. 2003 (2003), no. 48, 3067-3089.

S. V. Ludkovsky and A. Khrennikov, Stochastic processes on non-Archimedean spaces with values in non-Archimedean fields, Markov Process. Related Fields 9 (2003), no. 1, 131-162.

P. Malliavin, Stochastic Analysis, Grundlehren der Mathematischen Wissenschaften [Fundamental Principles of Mathematical Sciences], vol. 313, Springer-Verlag, Berlin, 1997.

H. P. Mc Kean, Stochastic Integrals, Izdat. "Mir”, Moscow, 1972 (Russian).

B. Øksendal, Stochastic Differential Equations, Universitext, Springer-Verlag, Berlin, 1995.

W. H. Schikhof, Ultrametric Calculus, Cambridge Studies in Advanced Mathematics, vol. 4, Cambridge University Press, Cambridge, 1984.

A. C. M. van Rooij, Non-Archimedean Functional Analysis, Monographs and Textbooks in Pure and Applied Math., vol. 51, Marcel Dekker, New York, 1978.

S. Vatanabè and N. Ikèda, Stochastic Differential Equations and Diffusion Processes, "Nauka", Moscow, 1986 (Russian).

0] V. S. Vladimirov, I. V. Volovich, and E. I. Zelenov, $p$-Adic Analysis and Mathematical Physics, VO "Nauka", Moscow, 1994.

41] K. Yasuda, Additive processes on local fields, J. Math. Sci. Univ. Tokyo 3 (1996), no. 3, 629654.

S. V. Ludkovsky: Department of Theoretical Researches, General Physics Institute, Russian Academy of Sciences, Street Vavilov 38, Moscow 119991 GSP-1, Russia

E-mail address: 7udkovsk@fpl.gpi .ru 


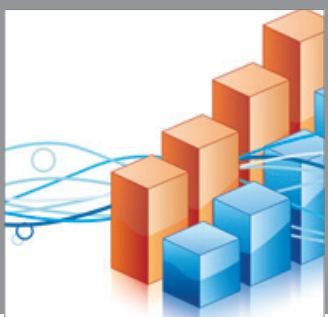

Advances in

Operations Research

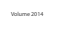

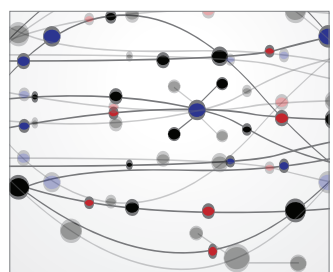

\section{The Scientific} World Journal
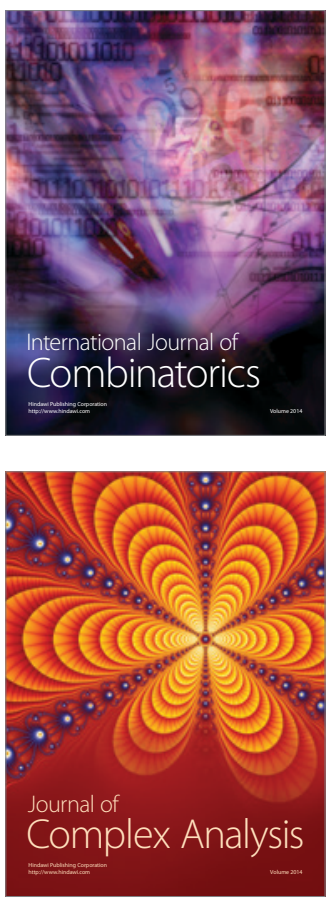

International Journal of

Mathematics and

Mathematical

Sciences
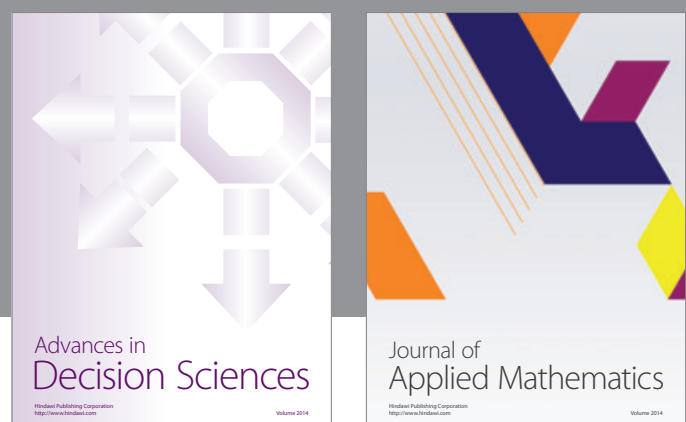

Journal of

Applied Mathematics
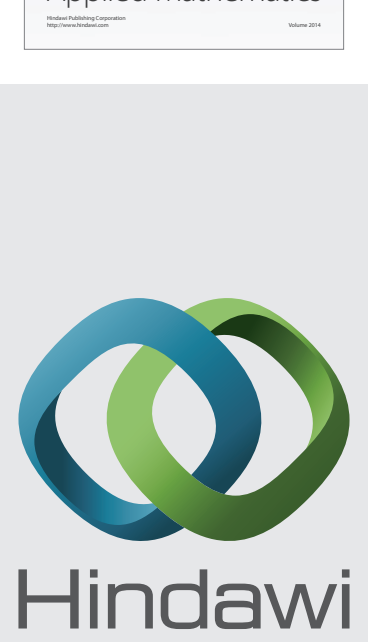

Submit your manuscripts at http://www.hindawi.com
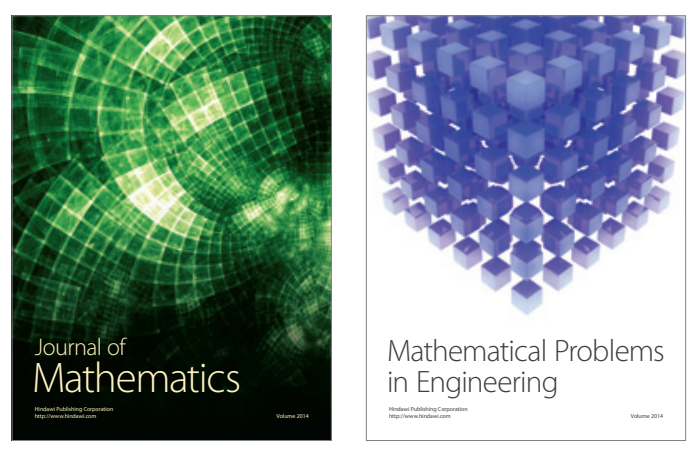

Mathematical Problems in Engineering
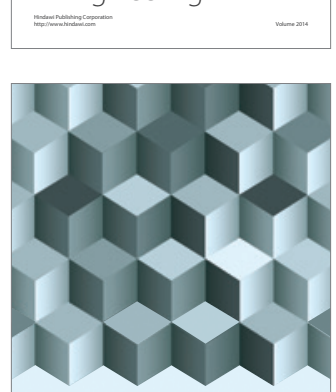

Journal of

Function Spaces
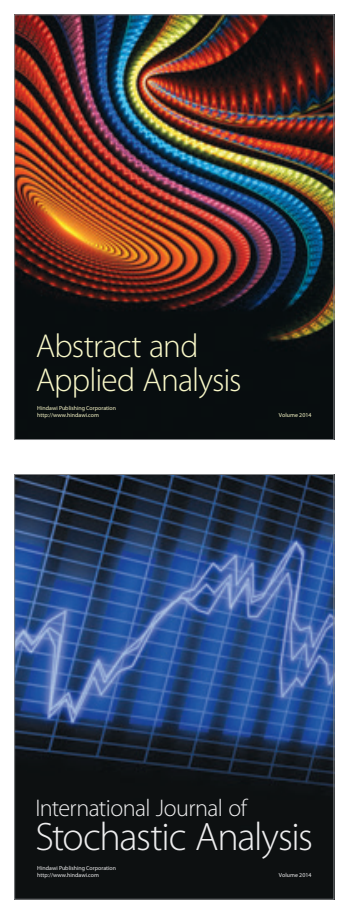

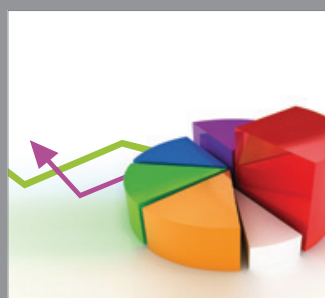

ournal of

Probability and Statistics

Promensencen
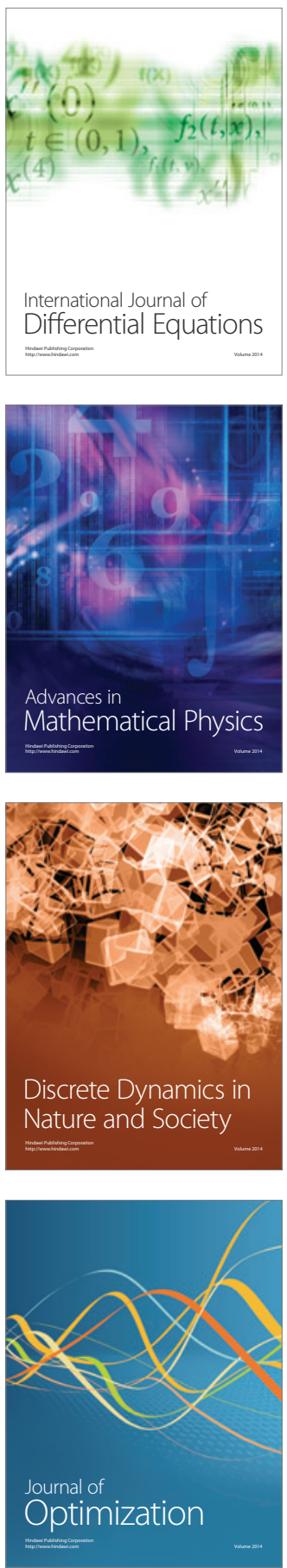